\title{
LA CARTELERA TEATRAL DE CHICLANA DE LA FRONTERA DURANTE EL SIGLO XIX. OBRAS, AUTORES, COMPAÑÍAS Y RECEPCIÓN CRÍTICA
}

\author{
THE THEATRE PROGRAMME IN CHICLANA DE LA FRONTERA \\ DURING THE NINETEENTH CENTURY. PLAYS, AUTHORS, \\ COMPANIES \\ AND CRITICAL RECEPTION
}

\section{Francisco ÁLVAREZ HORTIGOSA}

IES Poeta García Gutiérrez

hhortigosa@gmail.com

Resumen: Este artículo presenta la reconstrucción y análisis de la cartelera teatral de Chiclana de la Frontera durante el siglo XIX, la nómina de obras y autores llevados a escena, las compañías y actores que los representaron y la recepción crítica que se les dispensó.

Palabras clave: Cartelera teatral. Teatro español del Siglo XIX. Chiclana de la Frontera.

Abstract: This paper presents the reconstruction and analysis of the theatre programme in Chiclana de la Frontera during the nineteenth century, the list of authors and plays performed, the companies and actors performing them, as well as the reviews received. 
Key Words: Theatre programme. Spanish drama in the nineteenth century. Chiclana de la Frontera.

\section{INTRODUCCIÓN}

La escena teatral chiclanera del siglo XIX tuvo como centro fundamental el Teatro de Chiclana. Capaz de acoger a 1.000 personas a finales de los años cuarenta (Pascual Madoz, 1847: 322), pasó a disponer de 600 localidades en 1881 como resultado de un proceso de reformas que transformaron un edificio construido de madera en un recinto renovado con materiales más perdurables, primando en el número de asientos la comodidad frente a la cantidad. Permaneció activo al menos desde la década de 1820 hasta finales de los años 80 .

Si bien la villa solo alcanzó una población máxima de 12.339 habitantes en 1887, el público se veía incrementado durante la temporada estival con la afluencia de gaditanos de las clases acomodadas (Bohórquez Jiménez, 1996: 287).

La intervención de las autoridades, tanto municipales como provinciales, en el desarrollo de los espectáculos teatrales fue continua. Desde el Ayuntamiento se eligen los diputados de comedias y se proponen los nombres para el cargo de censor al Gobierno Civil de la Provincia, ante el que también se tramitan las licencias solicitadas por los representantes de las compañías para dar funciones en la ciudad.

\footnotetext{
${ }^{1}$ A.H.M.Ch. Secretaría. Registro, Correspondencia y Comunicaciones. Signatura 201. Estadística de los Edificios destinados a espectáculos públicos en esta Ciudad.
} 
Las fuentes consultadas en los archivos Histórico Municipal de Chiclana (A.H.M.Ch.) e Histórico Provincial de Cádiz (A.H.P.C.) proporcionan el grueso de la información tratada. La Biblioteca Municipal de Chiclana (B.M.Ch.) conserva ejemplares de La Semana (1930-31) y El Trovador (1932-33), publicaciones periódicas donde se recogen varias crónicas sobre la vida escénica local decimonónica2.

\section{CARTELERA TEATRAL DE CHICLANA DE LA FRONTERA DURANTE EL SIGLO XIX}

La cartelera teatral de Chiclana durante el siglo XIX se inicia en 1806 y concluye el 27 de octubre de 1889, con datos, además de para esos dos años, de 1830-40, 1849, 1850, 1877, 1879 y 1883. La conforman los locales, compañías, fechas en que se realizan las funciones y obras puestas en escena. Todas las actuaciones, salvo las llevadas a cabo en 1806 cuyo lugar de representación no hemos podido verificar, se efectuaron en el Teatro de Chiclana.

\footnotetext{
${ }^{2}$ Este trabajo sigue en gran medida la línea de investigación en torno a la vida escénica contemporánea española desarrollada desde el Centro de Investigación de Semiótica Literaria, Teatral y Nuevas Tecnologías (UNED), dirigido por el profesor José Romera Castillo, cuyas actividades pueden verse en "Estudios sobre teatro", http://www.uned.es/ centro-investigación-SELITENT@/estudios_sobre_teatro.html[28/04/2018]. Así como lo consignado en el fundamental volumen de José Romera Castillo (2011), Pautas para la investigación del teatro español y sus puestas en escena.
} 


\begin{tabular}{|c|c|c|}
\hline \multicolumn{3}{|c|}{ Número de funciones registradas distribuidas por años y compañías } \\
\hline $\begin{array}{c}\text { Fecha de } \\
\text { representación }\end{array}$ & $\begin{array}{l}\quad{ }^{\mathrm{o}}{ }^{\circ} \mathrm{de} \\
\text { funciones: } 80\end{array}$ & Compañía \\
\hline $\begin{array}{c}1806 \text { (Bohórquez } \\
\text { Jiménez, 2001: 52) } \\
\text { 1830-1840 }\end{array}$ & Sin determinar & Sin determinar \\
\hline $\begin{array}{l}\text { 1849. Junio, días 13, } \\
15 \text { (El Liro, 10-06- } \\
\text { 1849, n. } .^{\circ} \text {; 17-07- } \\
\left.1849, \text { n. }^{\circ} 3\right)\end{array}$ & 2 & $\begin{array}{c}\text { Compañía dramática, Sr. } \\
\text { Torromé }\end{array}$ \\
\hline $\begin{array}{l}\text { 1850. Agosto- } \\
\text { septiembre }^{5}\end{array}$ & 3 & $\begin{array}{l}\text { Compañía dramática dirigida } \\
\text { por Fernando González }\end{array}$ \\
\hline 1877. Julio, $29^{6}$ & 1 & $\begin{array}{c}\text { Compañía dirigida por Carlos } \\
\text { Barrilaro del Valle }\end{array}$ \\
\hline
\end{tabular}

${ }^{3}$ Según Frasquita Larrea en el año de 1806 se representaban obras como El mejor alcalde el rey, El rico hombre de Alcalá, El honor da entendimiento y "[...] otras piezas igualmente buenas".

${ }^{4}$ A.H.P.C., 1.3.8. Gobierno Civil, 2.4.32, caja 154, exp. 50. El apoderado del autor de la comedia Carlos II el Hechizado se queja contra la empresa del Teatro de Chiclana de la Frontera por el impago de los derechos de representación. 19.06.1846 - 18.07.1846. ${ }^{5}$ A.H.P.C., 1.3.8. Gobierno Civil, 2.4.32, caja 156, exp. 53. Fernando González solicita permiso para dar funciones dramáticas en Chiclana de la Frontera. 26.08.1850 06.09.1850.

${ }^{6}$ A.H.M.Ch. Secretaría. Registro, Correspondencia y Comunicaciones. Signatura 199. Se recoge en una nota la función del domingo 29 de julio de 1877 . 


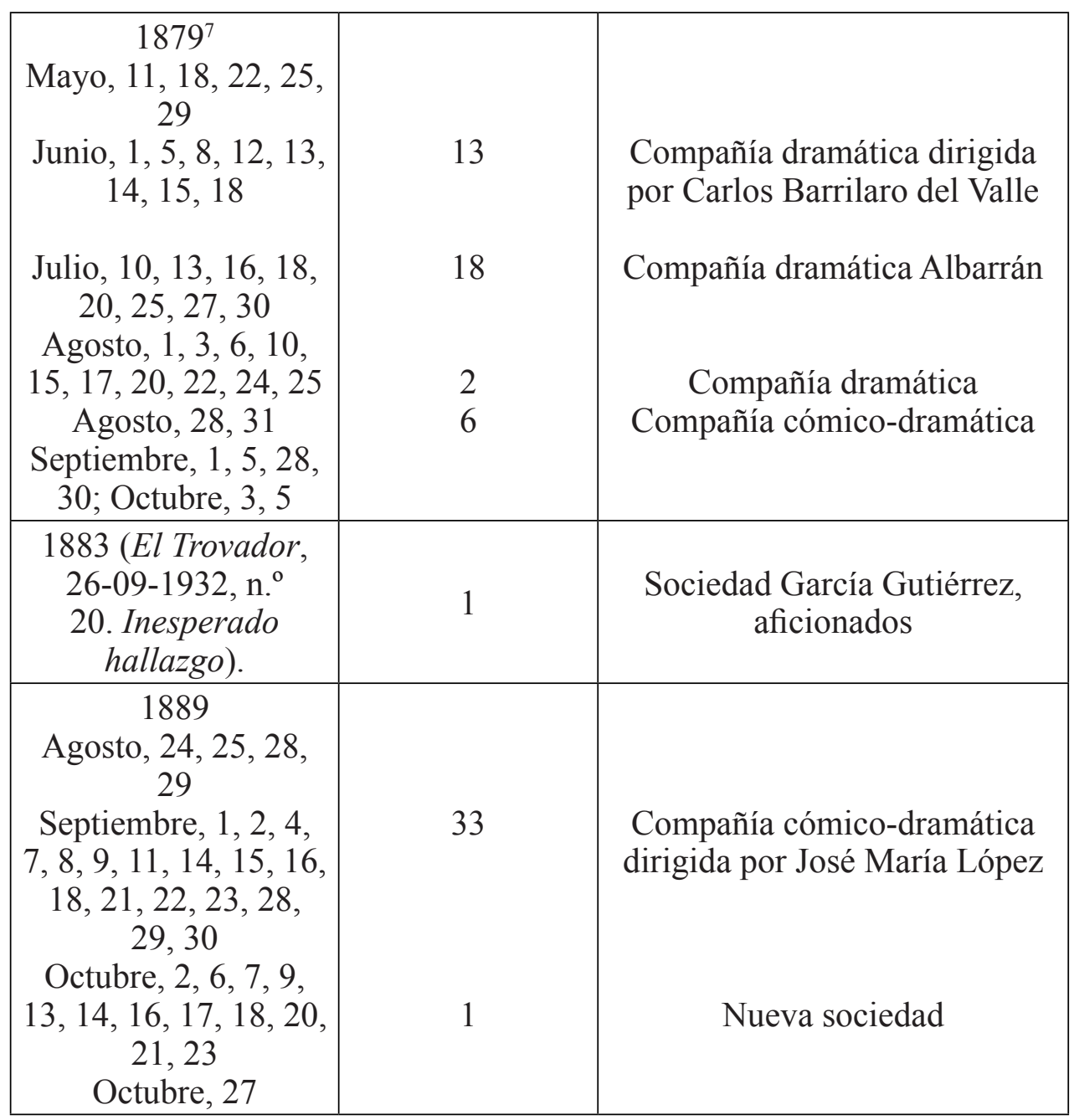

\footnotetext{
${ }^{7}$ A.H.M.Ch. Cultura. Programas de mano del Teatro de Chiclana. Gracias a estos programas conocemos la función del día 11-05-1879 y las comprendidas entre el 24-08-1889 y el 27-10-1889. A.H.M.Ch. Cultura. Festejos. Expedientes anuales. Signatura 858. Nota de las funciones de teatro. 1879. Funciones entre el 18-05-1879 y el 05-10-1879.
} 


\subsection{Relación de obras representadas en Chiclana de la Frontera}

El repertorio por orden alfabético de las obras que subieron a los escenarios chiclaneros incluye 125 títulos. De cada obra apuntamos las referencias aportadas por las fuentes investigadas en Chiclana, género, número de actos, escrita en prosa o verso, autor, compañía y su director, temporada, fecha y número de representaciones llevadas a cabo. Completamos la información a partir de otros trabajos sobre el teatro representado en la España del siglo XIX (Álvarez Hortigosa, 2012; Bajo Jiménez, Romero Ferrer, Vargas Zúñiga, 2002; Biblioteca Nacional, 1989, 1991; Fundación Juan March, 1986, 1993; http://www.uned.es/centroinvestigación-SELITENT@/estudios_sobre_teatro.html[28/03/2018]) $)^{8}$.

\section{Abreviaturas}

a. Acto

Ad. Adaptación

C. Comedia o Compañía

c. Cuadro

Ca. Caricatura

có. Cómico

cost. Costumbres

D. Drama o Director

Di. Disparate

dr. Dramático

F. Fecha

fi. Filosófico

G. Género

Gi. Gitanesco

Gr. Gracioso h. Humorístico

hist. Histórico

J. Juguete

Lo. Loa

Md. Melodrama

Pas. Pasillo

Pi. Pieza

pr. Prosa

pró. Prólogo

R. Número de representaciones

S. Sainete

T. Tragedia

Temp. Temporada

td. Traducción

vr. Verso

\footnotetext{
${ }^{8}$ En la elaboración del listado de obras seguimos la metodología empleada por Dougherty y Vilches de Frutos en su trabajo sobre la escena madrileña entre 1918 y 1926 (Dougherty y Vilches de Frutos, 1990: 161-491).
} 


\begin{tabular}{|c|c|}
\hline \multicolumn{2}{|c|}{ Listado de obras } \\
\hline $\begin{array}{l}\text { [1] ALCALDE TOREADOR (EL).- G: S. } \\
\text { Autor: Castillo, Juan del } \\
\text { C: Cómico-dramática } \\
\text { D: López, José M. } \\
\text { Temp. } 1889 \\
\text { F: 23-10-89.- R: } 1\end{array}$ & $\begin{array}{l}\text { [2] AMOR DE MADRE.- G: D. } 2 \text { a. } \\
\text { Autor: Desconocido } \\
\text { C: Cómico-dramática } \\
\text { D: López, José M. } \\
\text { Ad: Vega, Ventura de la } \\
\text { Temp. } 1889 \\
\text { F: } 18-09,07-10-89 .- \text { R: } 2\end{array}$ \\
\hline $\begin{array}{l}\text { [3] ANZUELO (EL).- G: C. } 3 \text { a., vr. } \\
\text { Autor: Blasco, Eusebio } \\
\text { C: Dramática Albarrán } \\
\text { D: Desconocido } \\
\text { Temp. } 1879 \\
\text { F: 22-08-79.- R: } 1\end{array}$ & $\begin{array}{l}\text { [4] BESO (EL).- G: C. } 1 \text { a., pr. } \\
\text { Autor: Infante de Palacios, Santiago } \\
\text { C: Cómico-dramática } \\
\text { D: López, José M. } \\
\text { Temp. } 1889 \\
\text { F: 28-08, 20-10-89.- R: } 2 \\
\end{array}$ \\
\hline $\begin{array}{l}\text { [5] CABANA DE TOM O LA } \\
\text { ESCLAVITUDDE LOS NEGROS (LA).- } \\
\text { G: D. cost. } 6 \text { a. } \\
\text { Autor: Valladares y Saavedra, Ramón de } \\
\text { C: Cómico-dramática } \\
\text { D: López, José M. } \\
\text { Temp. 1889 } \\
\text { F: 01-09, 16-09-89.- R: } 2\end{array}$ & $\begin{array}{l}\text { [6] CADA LOCO CON SU TEMA.- G: } \\
\text { C. } 2 \text { a., pr. } \\
\text { Autor: Ramos Carrión, Miguel } \\
\text { C: Dramática Albarrán } \\
\text { D: Desconocido } \\
\text { Temp. } 1879 \\
\text { F: } 15-08-79 .- \text { R: } 1\end{array}$ \\
\hline $\begin{array}{l}\text { [7] CAIGA EL QUE CAIGA.- G: J. có. } 1 \text { a. } \\
\text { Autor: Sánchez Castilla, Eduardo } \\
\text { C: Cómico-dramática } \\
\text { D: López, José M. } \\
\text { Temp. } 1889 \\
\text { F: 28-8-89.- R: } 1\end{array}$ & $\begin{array}{l}\text { [8] CAMINO DE PRESIDIO (EL).- G: D. } \\
\text { 6 a. } \\
\text { Autor: Ortiz de Pinedo, Manuel } \\
\text { C: Dramática } \\
\text { D: Barrilaro del Valle, Carlos } \\
\text { Temp. } 1879 \\
\text { F: } 18-05-79 .- \text { R: } 1\end{array}$ \\
\hline $\begin{array}{l}\text { [9] CAMPANILLA DE LOS APUROS } \\
\text { (LA).- G: J. có. } 1 \text { a. } \\
\text { Autor: Moreno Gil, Pantaleón } \\
\text { C: Dramática Albarrán } \\
\text { D: Desconocido } \\
\text { Temp. } 1879 \\
\text { F: } 10-07-79 ; 11-09,17-10-89 .- \text { R: } 3\end{array}$ & $\begin{array}{l}\text { [10] CAÑAS SE VUELVEN LANZAS } \\
\text { (LAS).- G: C. } 3 \text { a., vr. } \\
\text { Autor: García Gutiérrez, Antonio } \\
\text { C: Sociedad García Gutiérrez, aficionados } \\
\text { D: Desconocido } \\
\text { Temp. } 1883 \\
\text { F: Desconocida.- R: } 1\end{array}$ \\
\hline
\end{tabular}




\begin{tabular}{|l|l|}
\hline [11] CARETA VERDE (LA).- G: C. 2 a., & [12] ißCARIÑOS QUE MATAN!!.- G: C. \\
pr. & 3 a., vr. \\
Autor: Ramos Carrión, Miguel & Autor: Palencia, Ceferino \\
C: Dramática & C: Cómico-dramática \\
D: Barrilaro del Valle, Carlos & D: López, José M. \\
Temp. 1879 & Temp. Desconocidas \\
F: 29-05-79.- R: 1 & F: Décadas 1830-1840.- R: 1 \\
\hline [13] CARLOS II ELHECHIZADO.- G: D. & [14] CASA DE ABATES LOCOS (LA).- \\
5 a., vr. & G: S. \\
Autor: Gil de Zárate, Antonio & Autor: Desconocido \\
C: Desconocida & C: Cómico-dramática \\
D: Desconocido & D: López, José M. \\
Temp. 1889 & Temp. 1889 \\
F: 15-08-89.- R: Desconocidas & F: 02-09-89.- R: 1 \\
\hline [15] CASA DE FIERAS (UNA).- G: C. 1 & [16] CASA DE PRÉSTAMOS (UNA).- G: \\
a., pr. & Pas. fi. 1 a., vr. \\
Autor: Liern y Cerach, Rafael María & Autor: Jackson Veyan, José \\
C: Dramática Albarrán & C: Cómico-dramática \\
D: Desconocido & D: López, José M. \\
Temp. 1879 & Temp. 1889 \\
F: 01-08-79.- R: 1 & F: 24-08-89.- R: 1 \\
C: Cómico-dramática & \\
D: López, José M. & \\
Temp. 1889 & \\
F: 13-10-89.- R 1 & \\
\hline [17] CELOS DEL TÍO MACACO (LOS).- & [18] CITAS (LAS).- G: C. 1 a., pr. \\
G: C. 1 a., vr. & Autor: Desconocido \\
Autor: Sanz Pérez, José & C: Cómico-dramática \\
C: Dramática Albarrán & D: López, José M. \\
D: Desconocido & Temp. 1889 \\
Temp. 1879 & F: 16-10-89.- R: 1 \\
F: 25-08-79.- R: 1 & \\
\hline
\end{tabular}




\begin{tabular}{|c|c|}
\hline $\begin{array}{l}\text { [19] CÓMO EMPIEZA Y CÓMO } \\
\text { ACABA.- G: D. } 3 \text { a., vr. } \\
\text { Autor: Echegaray, José } \\
\text { C: Cómico-dramática } \\
\text { D: Desconocido } \\
\text { Temp. } 1879 \\
\text { F: 05-10-79.- R: } 1\end{array}$ & $\begin{array}{l}\text { TRA VIENTO Y I } \\
\text { egaray, Miguel } \\
\text { ca } \\
\text { o del Valle, Carlos }\end{array}$ \\
\hline $\begin{array}{l}\text { [21] CUARTO CON DOS CAMAS } \\
\text { G: J. có. } 1 \text { a. } \\
\text { Autor: Peral, Juan del } \\
\text { C: Dramática } \\
\text { D: Sr. Torromé } \\
\text { Temp. } 1849 \\
\text { F: } 13-06-49 .- \text { R: } 1\end{array}$ & $\begin{array}{l}\text { [22] DAR EN EL BLANCO.- G } \\
\text { vr. } \\
\text { Autor: Pina Domínguez, Mariano } \\
\text { C: Dramática Albarrán }\end{array}$ \\
\hline $\begin{array}{l}\text { [23] DE ASISTENTE A CAPITÁN.- G: } \\
\text { có. } 1 \text { a. } \\
\text { Autor: Mota González, José } \\
\text { C: Cómico-dramática } \\
\text { D: López, José M. } \\
\text { Temp. } 1889\end{array}$ & del Valle, Carlos \\
\hline $\begin{array}{l}\text { [25] DEL ENEMIGO EL CONSEJ } \\
\text { C. } 3 \text { a., vr. } \\
\text { Autor: Zamora Caballero, Eduardo } \\
\text { C: Cómico-dramática } \\
\text { D: López, José M. } \\
\text { Temp. } 1889\end{array}$ & $\begin{array}{l}\text { [26] DF } \\
\text { Autor: } \\
\text { C: Drar } \\
\text { D: Des } \\
\text { Temp. } \\
\text { F: } 24-0\end{array}$ \\
\hline
\end{tabular}




\begin{tabular}{|l|l|}
\hline [27] DIEGO CORRIENTES O EL & [28] DIEGO CORRIENTES (2a PARTE).- \\
BANDIDO GNEROSO.- G: D. 3 a., vr. & G: C. 3 a. \\
Autor: Gutiérrez de Alba, José María & Autor: Zumel, Enrique \\
C: Dramática Albarrán & C: Cómico-dramática \\
D: Desconocido & D: Desconocido \\
Temp. 1879 & Temp. 1879 \\
F: 25-08-79.- R: 1 & F: 01-09-79.- R: 1 \\
C: Cómico-dramática & \\
D: López, José M. & \\
Temp. 1889 & \\
F: 21-10-89.- R: 1 & \\
\hline [29] DOCE RETRATOS, SEIS REALES.- & [30] DOMINÓS BLANCOS (LOS).- G: C. \\
G: C. 1 a. & 3 a., pr. \\
Autor: Ramos Carrión, Miguel & Autor: Desconocido \\
C: Dramática Albarrán & C: Dramática Albarrán \\
D: Desconocido & D: Desconocido \\
Temp. 1879 & Ad: Navarrete, Ramón de; Pina Domínguez, \\
F: 18-07, 20-08-79.- R: 2 & Mariano \\
& Temp. 1879 \\
\hline [31] D. RICARDO Y D. RAMÓN.- G: Pi. & [32] DOÑA JUANA TENORIO.- G: Pi. 1 \\
1 a. & a., vr. \\
Autor: Desconocido & Autor: Liern y Cerach, Rafael María \\
C: Dramática & C: Cómico-dramática \\
D: Barrilaro del Valle, Carlos & D: López, José M. \\
Temp. 1879 & Temp. 1889 \\
F: 01-06-79.- R: 1 & F: 24-08-89.- R: 1 \\
\hline [33] DOS CONTRA UNO O LOS & [34] DOS HIJOS O EL TRIBUTO DE LA \\
ENREDOS DE UNA VIUDA.- G: Pi. 1 a. & SANGRE.- G: D. 1 a., vr. \\
Autor: Desconocido & Autor: Fernández Bremón, José \\
C: Dramática & C: Cómico-dramática \\
D: Sr. Torromé & D: López, José M. \\
Temp. 1849 & Temp. 1889 \\
F: 15-06-49.- R: 1 & F: 28-08, 09-09-89.- R: 2 \\
\hline
\end{tabular}




\begin{tabular}{l} 
[35] DOS JOYAS DE LA CASA (LAS).- G: \\
C. 1 a., pr. \\
Autor: Corzo y Barrera, Antonio \\
C: Dramática \\
D: Desconocido \\
Temp. 1879 \\
F: 28-08-79.- R: 1 \\
\hline [37] DOS Y UNO.- G: C. 1 a. \\
Autor: Bueno de Saucal, Ignacio María; \\
Sánchez Garay, Laureano \\
C: Dramática Albarrán \\
D: Desconocido \\
Temp. 1879 \\
F: 16-07-79.- R: 1
\end{tabular}

[39] EL QUE VA PARA OCHAVO.- G: C. 1 a.

Autor: Echegaray, Miguel

C: Cómico-dramática

D: Desconocido

Temp. 1879

F: 03-10-79.- R: 1

[41] EQUILIBRIO EUROPEO (EL).- G:

\section{2 a., pr.}

Autor: Sánchez de Castilla, Eduardo; Gómez de Cádiz, Manuel

C: Dramática Albarrán

D: Desconocido

Temp. 1879

F: 06-08-79.- R: 1

[43] ESPOSA DEL VENGADOR (LA).- G:

D. 3 a., vr.

Autor: Echegaray, José

C: Dramática

D: Barrilaro del Valle, Carlos

Temp. 1877

F: 29-07-77.- R: 1
[36] DOS MUERTOS Y NINGÚN DIFUNTO.- G: C. 2 a.
Autor: Mélesville; Pinel Dumanoir, François
C: Dramática Albarrán
D: Desconocido
Ad: Coll, Fernando
Temp. 1879
F: 18-07-79.- R: 1

[38] ECHAR LA LLAVE.- G: C. 1 a., vr.

Autor: Echegaray, Miguel

C: Dramática

D: Desconocido

Temp. 1879

F: 31-08-79.- R: 1

[40] EN PLENA LUNA DE MIEL.- G: C.

1 a., vr.

Autor: Echegaray, Miguel

C: Cómico-dramática

D: López, José M.

Temp. 1889

F: 18-09-89.- R: 1

[42] ESCLAVO DE SU CULPA (EL).- G:

D. 3 a., vr.

Autor: Cavestany, Juan Antonio

C: Dramática

D: Barrilaro del Valle, Carlos

Temp. 1879

F: 12-06, 18-06-79.- R: 2

[44] FERIA DE SAN ANTONIO DE CHICLANA (LA).- G: Pi. 1 a.

Autor: Desconocido

C: Dramática Albarrán

D: Desconocido

Temp. 1879

F: 14-06-79.- R: 1 


\begin{tabular}{|c|c|}
\hline $\begin{array}{l}\text { [45] FOGÓN Y EL MINISTERIO (EL).- } \\
\text { G: C. } 3 \text { a., pr. } \\
\text { Autor: Pina Domínguez, Mariano } \\
\text { C: Dramática Albarrán } \\
\text { D: Desconocido } \\
\text { Temp. } 1879 \\
\text { F: } 30-07-79 .- \text { R: } 1\end{array}$ & $\begin{array}{l}\text { [46] FORASTERO (EL).- G: C. } 3 \text { a. } \\
\text { Autor: Pina Domínguez, Mariano } \\
\text { C: Dramática Albarrán } \\
\text { D: Desconocido } \\
\text { Temp. } 1879 \\
\text { F: 20-08-79.- R: } 1\end{array}$ \\
\hline $\begin{array}{l}\text { [47] ¡FUERZA DE UN NIÑO (LA)!.- G: C. } \\
\mathbf{3} \text { a., vr. } \\
\text { Autor: Echegaray, Miguel } \\
\text { C: Cómico-dramática } \\
\text { D: López, José M. } \\
\text { Temp. } 1889 \\
\text { F: } 29-08-89 .- \text { R: } 1\end{array}$ & $\begin{array}{l}\text { [48] GUERRA EN CALZONCILLOS } \\
\text { (LA).- G: C. } 1 \text { a. } \\
\text { Autor: Sánchez Albarrán, José } \\
\text { C: Dramática Albarrán } \\
\text { D: Desconocido } \\
\text { Temp. } 1879 \\
\text { F: 22-08-79.- R: } 1\end{array}$ \\
\hline $\begin{array}{l}\text { [49] GUERRAS CIVILES (LAS).- G: D. } 3 \\
\text { a., vr. } \\
\text { Autor: Asquerino, Eduardo; Asquerino, } \\
\text { Eusebio } \\
\text { C: Dramática } \\
\text { D: Desconocido } \\
\text { Temp. } 1849 \\
\text { F: } 15-06-49 .- \text { R: } 1\end{array}$ & $\begin{array}{l}\text { [50] HIJAS DE ELENA (LAS).- G: C. } 1 \\
\text { a., vr. } \\
\text { Autor: García Santisteban, Rafael } \\
\text { C: Cómico-dramática } \\
\text { D: López, José M. } \\
\text { Temp. } 1889 \\
\text { F: } 11-09-89 .- \text { R: } 1\end{array}$ \\
\hline $\begin{array}{l}\text { [51] HONOR DA ENTENDIMIENTO } \\
\text { (EL).- G: C. } \\
\text { Autor: Cañizares, José de } \\
\text { C: Desconocida } \\
\text { D: Desconocido } \\
\text { Temp. } 1806 \\
\text { F: Desconocida.-R: Desconocidas }\end{array}$ & $\begin{array}{l}\text { [52] HUÉSPED DEL OTRO MUNDO } \\
\text { (UN).- G: C. } 1 \text { a., vr. } \\
\text { Autor: Serra, Narciso } \\
\text { C: Dramática Albarrán } \\
\text { D: Desconocido } \\
\text { Temp. } 1879 \\
\text { F: } 31-08-79 .- \text { R: } 1\end{array}$ \\
\hline $\begin{array}{l}\text { [53] INDEPENDENCIA (LA).- G: C. } 4 \text { a. } \\
\text { Autor: Bretón de los Herreros, Manuel } \\
\text { C: Dramática Albarrán } \\
\text { D: Desconocido } \\
\text { Temp. } 1879 \\
\text { F: } 15-08-79 .- \text { R: } 1\end{array}$ & $\begin{array}{l}\text { [54] INOCENCIA.- G: C. } 3 \text { a., vr. } \\
\text { Autor: Echegaray, Miguel } \\
\text { C: Dramática } \\
\text { D: Barrilaro del Valle, Carlos } \\
\text { Temp. } 1879 \\
\text { F: 13-06-79.- R: } 1\end{array}$ \\
\hline
\end{tabular}


[55] JUAN EL PERDÍO.- G: Di. h., vr.

Autor: Mejías y Escassy, Luis

C: Cómico-dramática

D: Desconocido

Temp. 1879

F: 30-09-79.- R: 1

[59] ¡LANCEROS!.- G: J. có. 1 ac., vr.

Autor: Chacel, Mariano

C: Cómico-dramática

D: López, José M.

Temp. 1889

F: 22-09-89.- R: 1
[56] ¡JUEZ Y PARTE!.- G: J. có. 1 a.

Autor: Mínguez, Federico; Rubio, Ángel

C: Cómico-dramática

D: López, José M.

Temp. 1889

F: 04-09-89.- R: 1

[60] ¡LEVANTAR MUERTOS!.- G: C. 2 a., pr.

Autor: Blasco, Eusebio; Ramos Carrión, Miguel

C: Dramática

D: Desconocido

Temp. 1879

F: 31-08-79.- R: 1

Cómico-dramática

D: López, José M.

Temp. 1889

F: 07-09, 30-09-89.- R: 2

[62] ¡LO QUE VALE EL TALENTO!.- G:

C. 3 a.

Autor: Pérez Echevarría, Francisco

C: Cómico-dramática

D: López, José M.

Temp. 1889

F: 22-09, 17-10-89.- R: 2

F: $22-09,17-10-89 .-\mathrm{R}: 2$

[64] LLOVIDO DEL CIELO.- G: C. 2 a., vr.

Autor: Aza, Vital

C: Dramática Albarrán

D: Desconocido

Temp. 1879

F: 10-07-79.- R: 1 


\begin{tabular}{|l|l|}
\hline [65] LLUEVEN HIJOS.- G: J. có. 1 a., pr. & [66] MAESTRO DE ESCUELA (EL).- G: \\
Autor: Antonio Bermejo, Ildefonso & Ca. \\
C: Dramática Albarrán & Autor: Peral, Juan del \\
D: Desconocido & C: Cómico-dramática \\
Temp. 1879 & D: López, José M. \\
F: 27-07-79.- R: 1 & Temp. 1889 \\
& F: 30-09-89.- R: 1 \\
\hline [67] MANSIÓN DEL CRIMEN (LA).- G: & [68] MARINOS EN TIERRA.- G: C. 1 a. \\
C. 1 a. & Autor: Sanz Pérez, José \\
Autor: Bretón de los Herreros, Manuel & C: Dramática Albarrán \\
C: Dramática Albarrán & D: Desconocido \\
D: Desconocido & Temp. 1879 \\
Temp. 1879 & F: 03-08-79.- R: 1 \\
F: 06-08-79.- R: 1 & C: Cómico-dramática \\
& D: López, José M. \\
& Temp. 1889 \\
\hline [69] ¡MARTES DE LAS DE GÓMEZ & F: 30-09-89.- R: 1 \\
(LOS)!.- G: Ca. 1 a., pr. & Autor: Desconocido \\
Autor: Barranco, Mariano & C: Dramática \\
C: Cómico-dramática & D: Barrilaro del Valle, Carlos \\
D: López, José M. & td: Olona, Luis \\
Temp. 1889 & Temp. 1877 \\
F: 11-09, 18-09-89.- R: 2 & F: 29-07-77.- R: 1 \\
\hline [71] ¡MARUJA!.- G: Pi., pr. & [72] MEJOR ALCALDE EL REY (EL).- \\
Autor: Lombía, Juan & G: C. 3 a., vr. \\
C: Cómico-dramática & Autor: Vega, Lope Félix de \\
D: López, José M. & C: Desconocida \\
Temp. 1889 & D: Desconocido \\
F: 07-09-89.- R: 1 & Temp. 1806 \\
& F: Desconocida.- R: Desconocidas \\
\hline
\end{tabular}




\begin{tabular}{|l|l|}
\hline [73] MEMORIALISTA (EL).- G: C. 2 a. & [74] MIÉRCOLES DE LA CRUZ O LAS \\
Autor: Olona, Luis & CURSIS BURLADAS (LOS).- G: S. 1 a. \\
C: Dramática Albarrán & Autor: Burgos, Javier de \\
D: Desconocido & C: Cómico-dramática \\
Temp. 1879 & D: López, José M. \\
F: 01-08-79.- R: 1 & Temp. 1889 \\
& F: 09-10-89.- R: 1 \\
\hline [75] MUJER DE ULISES (LA).- G: C. 1 & [76] NERVIOS DE MI MUJER (LOS).- \\
a., vr. & G: C. 1 a. \\
Autor: Blasco, Eusebio & Autor: Trigo, Carlos \\
C: Dramática Albarrán & C: Cómico-dramática \\
D: Desconocido & D: Desconocido \\
Temp. 1879 & Temp. 1879 \\
F: 25-07-79.- R: 1 & F: 28-09-79.- R: 1 \\
\hline [77] NO LA HAGAS Y NO LA TEMAS.- & [78] NOVENO MANDAMIENTO (EL).- \\
G: C. 2 a. & G: C. 3 a., pr. \\
Autor: Blasco, Eusebio & Autor: Ramos Carrión, Miguel \\
C: Cómico-dramática & C: Dramática \\
D: Desconocido & D: Barrilaro del Valle, Carlos \\
Temp. 1879 & Temp. 1879 \\
F: 28-09-79.- R: 1 & F: 05-06-79.- R: 1 \\
\hline [79] NOVIO DE DOÑA INÉS (EL).- G: Pi. & [80] NUDO GORDIANO (EL).- G: D. 3 a., \\
1 a., pr. y vr. & vr. \\
Autor: Burgos, Javier de & Autor: Sellés, Eugenio \\
C: Cómico-dramática & C: Dramática \\
D: López, José M. & D: Barrilaro del Valle, Carlos \\
Temp. 1889 & Temp. 1879 \\
F: 14-09-89.- R: 1 & F: 11-05-79.- R: 1 \\
\hline [81] ORACIÓN DE LA TARDE (LA).- G: & [82] OTRO GALLO LE CANTARA.- G: \\
Md. 3 a., pr. & C. 3 a., vr. \\
Autor: Larra, Luis Mariano de & Autor: Zumel, Enrique \\
C: Cómico-dramática & C: Dramática Albarrán \\
D: López, José M. & D: Desconocido \\
Temp. 1889 & Temp. 1879 \\
F: 16-10, 23-10-89.- R: & F: 13-07-79.- R: 1 \\
\hline & \\
\hline
\end{tabular}




\begin{tabular}{|l|l|}
\hline [83] PADRÓN MUNICIPAL (EL).- G: C. & [84] ¡PALOS DESEADOS (LOS)!.- G: S. \\
3 a., pr. & Autor: Cruz, Ramón de la \\
Autor: Ramos Carrión, Miguel; Aza, Vital & C: Cómico-dramática \\
C: Cómico-dramática & D: López, José M. \\
D: López, José M. & Temp. 1889 \\
Temp. 1889 & F: 09-09-89.- R: 1 \\
F: 04-09, 09-09-89.- R: 2 & \\
\hline [85] iPAÑO DE LÁGRIMAS (EL)! (L'AJO & [86] PARA MENTIR LAS MUJERES.- \\
NELL'IMBARAZZO).- G: C. 2 a., pr. & G: C. 1 a., pr. \\
Autor: Giraud, Giovanni & Autor: Calvacho, Carlos \\
C: Cómico-dramática & C: Dramática \\
D: López, José M. & D: Barrilaro del Valle, Carlos \\
Ad: Coello Pacheco, Carlos; Campo Arana, & Temp. 1879 \\
José & F: 11-05, 13-06-79.- R: 2 \\
Temp. 1889 & C: Cómico-dramática \\
F: 21-09-89.- R: 1 & D: López, José M. \\
& Temp. 1889 \\
\hline [87] PARTO DE LOS MONTES (EL).- G: & F: 24-08-89.- R: 1 \\
T. Gi. 2 a. & Autor: Cano y Masas, Leopoldo \\
Autor: Sanz Pérez, José & C: Cómico-dramática \\
C: Cómico-dramática & D: López, José M. \\
D: Desconocido & Temp. 1889 \\
Temp. 1879 & F: 25-08, 02-09-89.- R: 2 \\
F: 01-09-79.- R: 1 & \\
\hline [89] PAYO DE LA CARTA (EL).- G: S. & [90] PAYO EN CENTINELA (EL).- G: S. \\
Autor: Cruz, Ramón de la & 1 a. \\
C: Cómico-dramática & Autor: Ruíz Pelayo, Samuel \\
D: López, José M. & C: Sociedad García Gutiérrez, aficionados \\
Temp. 1889 & D: Desconocido \\
F: 23-09-89.- R: 1 & Temp. 1883 \\
& F: Desconocida.- R: Desconocidas \\
\hline
\end{tabular}




\begin{tabular}{|c|c|}
\hline $\begin{array}{l}\text { [91] PENAS DEL PURGATORIO (LAS).- } \\
\text { G: C. } 3 \text { a., pr. } \\
\text { Autor: Campo Arana, José; Fuentes, José } \\
\text { C: Dramática Albarrán } \\
\text { D: Desconocido } \\
\text { Temp. } 1879 \\
\text { F: 25-07-79.- R: } 1\end{array}$ & $\begin{array}{l}\text { [92] PEPITA.- G: C. } 1 \text { a. } \\
\text { Autor: Moro Morales, Emilio } \\
\text { C: Cómico-dramática } \\
\text { D: Desconocido } \\
\text { Temp. } 1879 \\
\text { F: 05-10-79.- R: } 1\end{array}$ \\
\hline $\begin{array}{l}\text { [93] PERRO DEL CAPITÁN (EL).- G: C. } \\
\mathbf{1} \text { a. } \\
\text { Autor: Vega, Ricardo de la } \\
\text { C: Dramática Albarrán } \\
\text { D: Desconocido } \\
\text { Temp. } 1879 \\
\text { F: } 13-07-79 .- \text { R: } 1\end{array}$ & $\begin{array}{l}\text { [94] PESQUISAS DE PATRICIO (LAS).- } \\
\text { G: C. } 3 \text { a. } \\
\text { Autor: Corona Bustamante, José; Villa y } \\
\text { Valle, J. de la } \\
\text { C: Dramática Albarrán } \\
\text { D: Desconocido } \\
\text { Temp. } 1879 \\
\text { F: 03-07-79.- R: } 1 \\
\end{array}$ \\
\hline $\begin{array}{l}\text { [95] PILLUELO DE PARÍS (EL).- G: D. } \\
\text { 2 a. } \\
\text { Autor: Bayard, Jean François Alfred; } \\
\text { Vanderbuch, Emile } \\
\text { C: Cómico-dramática } \\
\text { D: López, José M. } \\
\text { td: Lombía, Juan } \\
\text { Temp. } 1889 \\
\text { F: } 02-10,20-10-89 .- \text { R: } 2\end{array}$ & $\begin{array}{l}\text { [96] POBRE PORFIADO.- G: C. } 1 \text { a., vr. } \\
\text { Autor: Blasco, Eusebio } \\
\text { C: Cómico-dramática } \\
\text { D: Desconocido } \\
\text { Temp. } 1879 \\
\text { F: 28-09-79.- R: } 1\end{array}$ \\
\hline $\begin{array}{l}\text { [97] POBRES DE MADRID (LOS).- G: D. } \\
\text { cost. } 6 \text { c. y } 1 \text { pró. } \\
\text { Autor: Ortiz de Pinedo, Manuel } \\
\text { C: Cómico-dramática } \\
\text { D: López, José M. } \\
\text { Temp. } 1889 \\
\text { F: 08-09-89.- R: } 1\end{array}$ & $\begin{array}{l}\text { [98] PREMIO DEL BIEN (EL).- G: D. } 1 \text { a. } \\
\text { Autor: Desconocido } \\
\text { C: Dramática } \\
\text { D: Barrilaro del Valle, Carlos } \\
\text { Temp. } 1879 \\
\text { F: 05-06, 15-06-79.- R: } 2\end{array}$ \\
\hline
\end{tabular}




\begin{tabular}{|l|l}
\hline [99] PRIMO Y EL RELICARIO (EL).- G: & [100] PRUEBA DE AMOR \\
C. 3 a. & Autor: Jackson Veyan, José \\
Autor: Olona, Luis & C: Cómico-dramática \\
C: Dramática Albarrán & D: López, José M. \\
D: Desconocido & Temp. 1889 \\
Temp. 1879 & F: 28-09-89.- R: 1 \\
F: 20-07-79.- R: 1 &
\end{tabular}

[101] PRUEBA DEL SUBMARINO PERAL (LA).- G: Lo. dr. 1 a., vr.

Autor: Canales, Pedro

C: Cómico-dramática

D: López, José M.

Temp. 1889

F: 02-10-89.- R: 1
[102] REDIMIR AL CAUTIVO.- G: C. 3 a., vr.

Autor: Pina y Bohigas, Mariano

C: Dramática Albarrán

D: Desconocido

Temp. 1879

F: 30-07-79.- R: 1
[103] RICO HOMBRE DE ALCALÁ (EL).- G: C.

Autor: Moreto, Agustín

C: Desconocida

D: Desconocido

Temp. 1806

F: Desconocida.- R: Desconocidas
[104] ROBO EN DESPOBLADO.- G: C. 2 a., pr.

Autor: Ramos Carrión, Miguel; Aza, Vital C: Cómico-dramática

D: López, José M.

Temp. 1889

F: 28-09-89.- R: 1

[105] ROSA AMARILLA (LA).- G: C. 3 a., vr.

Autor: Blasco, Eusebio

C: Dramática

D: Barrilaro del Valle, Carlos

Temp. 1879

F: 25-05, 15-06, 03-10-79.- R: 3

[107] SEGUIDILLAS.- G: Pi. Gr. 1 a.

Autor: Sánchez de Castilla, Eduardo

C: Cómico-dramática

D: López, José M.

Temp. 1889

F: 06-10-89.- R: 1
[106] SAN SEBASTIÁN MÁRTIR.- G: C.

3 a.

Autor: Aza, Vital

C: Cómico-dramática

D: López, José M.

Temp. 1889

F: 09-10, 14-10-89.- R: 2

[108] SEPULTURERO
CEMENTERIO DE SAN NICOLÁS
(EL). G: D. 5 a., pr.
Autor: Mejías y Escassy, Luis
C: Cómico-dramática
D: López, José M.
Temp. 1889
F: 29-09, 18-10-89.- R: 2




\begin{tabular}{|c|c|}
\hline $\begin{array}{l}\text { [109] SERÁ ESTE.- G: Pi. } 1 \text { a. } \\
\text { Autor: Desconocido } \\
\text { C: Dramática } \\
\text { D: Barrilaro del Valle, Carlos } \\
\text { Temp. } 1879 \\
\text { F: } 18-06-79 .- \text { R: } 1\end{array}$ & $\begin{array}{l}\text { [110] ;SIN COCINERA!.- G: C. } 1 \text { a. } \\
\text { Autor: Matoses, Manuel } \\
\text { C: Cómico-dramática } \\
\text { D: López, José M. } \\
\text { Temp. } 1889 \\
\text { F: 21-09-89.- R: } 1 \\
\end{array}$ \\
\hline $\begin{array}{l}\text { [111] SOMBRA DE LA VENGANZA } \\
\text { (LA).- G: D. } 1 \text { a. } \\
\text { Autor: Desconocido } \\
\text { C: Dramática } \\
\text { D: Barrilaro del Valle, Carlos } \\
\text { Temp. } 1879 \\
\text { F: 29-05-79.- R: } 1\end{array}$ & $\begin{array}{l}\text { [112] SOMBRA NEGRA(LA).- G: Pi. } 1 \text { a., } \\
\text { pr. } \\
\text { Autor: Jackson Cortés, Eduardo } \\
\text { C: Dramática } \\
\text { D: Barrilaro del Valle, Carlos } \\
\text { Temp. } 1879 \\
\text { F: 25-05-79.- R: } 1\end{array}$ \\
\hline $\begin{array}{l}\text { [113] SOMBRERO DE COPA (EL).- G: C. } \\
\mathbf{3} \text { a., pr. } \\
\text { Autor: Aza, Vital } \\
\text { C: Cómico-dramática } \\
\text { D: López, José M. } \\
\text { Temp. } 1889 \\
\text { F: } 14-09,23-09-89 .- \text { R: } 2\end{array}$ & $\begin{array}{l}\text { [114] SOTA DE BASTOS (LA).- G: J. } 1 \\
\text { a., pr. } \\
\text { Autor: Fuentes, José de; Alcón, Aurelio } \\
\text { C: Dramática } \\
\text { D: Barrilaro del Valle, Carlos } \\
\text { Temp. 1879 } \\
\text { F: 29-05-79.- R: 1 } \\
\text { C: Dramática Albarrán } \\
\text { D: Desconocido } \\
\text { Temp. } 1879 \\
\text { F: 20-07-79.- R: 1 } \\
\text { C: Cómico-dramática } \\
\text { D: López, José M. } \\
\text { Temp. 1889 } \\
\text { F: 25-08-89.- R: } 1 \\
\end{array}$ \\
\hline $\begin{array}{l}\text { [115] SUEÑO DE UN MALVADO O EL } \\
\text { ASESINO DE GERMÁN (EL).- G: D. } 3 \text { a. } \\
\text { Autor: Hugo, Víctor } \\
\text { C: Dramática } \\
\text { D: Barrilaro del Valle, Carlos } \\
\text { Temp. } 1879 \\
\text { F: } 14-06-79 .- \text { R: } 1\end{array}$ & $\begin{array}{l}\text { [116] SUMA Y SIGUE.- G: Pi. } 1 \text { a., pr. } \\
\text { Autor: Pina y Bohigas, Mariano } \\
\text { C: Cómico-dramática } \\
\text { D: López, José M. } \\
\text { Temp. } 1889 \\
\text { F: 29-08-89.- R: } 1\end{array}$ \\
\hline
\end{tabular}


[117] TERREMOTO DE LA MARTINICA (EL).- G: D. 4 a. y 1 pró.

Autor: Cruz Tirado y Nario, Juan de la; Coll, Gaspar Fernando

C: Dramática Albarrán

D: Desconocido

Temp. 1879

F: 10-08-79.- R: 1

[119] TRAIDOR, INCONFESO Y MÁRTIR.- G: D. hist. 3a., vr.

Autor: Zorrilla, José

C: Dramática

D: Sr. Torromé

Temp. 1849

F: 13-06-49.- R: 1
[118] TIGRE DE BENGALA (EL).- G: Pi. 1 a.

Autor: Valladares y Saavedra, Ramón de

C: Dramática

D: Barrilaro del Valle, Carlos

Temp. 1879

F: 08-06-79.- R: 1

[120] TRAPERO DE MADRID (EL).- G:

D. 4 a. y 1 pró.

Autor: Desconocido

C: Dramática

D: Barrilaro del Valle, Carlos

Ad: Lombía, Juan

Temp. 1879

F: 22-05-79.- R: 1

[121] TRAPOS DE CRISTIANAR O LA GUERRA EN EL HOGAR (LOS).- G: C.

Gr. 3 a., pr.

Autor: Campo Arana, José; Estremera, José

C: Nueva Sociedad

D: Desconocido

Temp. 1889

F: 27-10-89.- R: 1

[123] TROVADOR (EL).- G: D. 5 a., pr. y

vr.

Autor: García Gutiérrez, Antonio

C: Cómico-dramática

D: Desconocido

Temp. 1879

F: 05-09-79.- R: 1

[122] TRAVESURAS DE JUANA (LAS).G: C. 4 a., vr.

Autor: García Doncel, Carlos; Valladares, Luis

C: Dramática Albarrán

D: Desconocido

Temp. 1879

F: 17-08-79.- R: 1

[124] VECINO DE AHÍ AL LADO (EL).-

G: J. cóm. 1 a., pr.

Autor: Gil y Luengo, Constantino

C: Nueva Sociedad

D: Desconocido

Temp. 1889

F: 27-10-89.- R: 1

\section{[125] VECINO DE ENFRENTE (EL).- G:}

J. có. 1 a., vr.

Autor: Blasco, Eusebio

C: Dramática Albarrán

D: Desconocido

Temp. 1879

F: 24-08-79.- R: 1 


\begin{tabular}{|c|c|c|c|c|c|c|c|c|c|c|}
\hline \multirow[b]{2}{*}{ Ginaro } & \multicolumn{9}{|c|}{ Total de obras presentadas en Cbiclaua por géneto y número de actos } & \multirow[b]{2}{*}{ Tot:l } \\
\hline & $\begin{array}{c}1 \\
\text { ivelu }\end{array}$ & $\begin{array}{c}2 \\
2: i k 1 \times\end{array}$ & $\stackrel{2}{a x i b s}$ & $\begin{array}{c}4 \\
a x \cdot 13 x\end{array}$ & $\begin{array}{c}5 \\
5 \\
\text { axtax }\end{array}$ & $\begin{array}{c}6 \\
\text { 2:ilex }\end{array}$ & $\begin{array}{c}6 \\
\text { تLstirns }\end{array}$ & $\begin{array}{c}10 \\
\text { cuaskux }\end{array}$ & $\begin{array}{l}\text { Sir. } \\
\text { dret:r- } \\
\text { mingr }\end{array}$ & \\
\hline Cosucdis. & 26 & 9 & 28 & 2 & & & & & 1 & 67 \\
\hline Exicatutu & $\mathrm{L}$ & & & & & & & & I & 2 \\
\hline $\begin{array}{c}\text { Dispurste } \\
\text { hums:ristical }\end{array}$ & & & & & & & & & 1 & 1 \\
\hline . & 1 & & & & & & & & & 1 \\
\hline $\begin{array}{l}\text { Juzatete } \\
\text { cồni: }\end{array}$ & 9 & & & & & & & & & 9 \\
\hline $\begin{array}{l}\text { Pasille } \\
\text { fil rentico }\end{array}$ & 1 & & & & & & & & & 1 \\
\hline Pies: & 10 & & & & & & & & 1 & 11 \\
\hline $\begin{array}{c}\text { Picra } \\
\text { cómina: }\end{array}$ & 1 & & & & & & & & & 1 \\
\hline Sain:ce & $\underline{z}$ & & & & & & & & 4 & 6 \\
\hline Daunta & 2 & 3 & 10 & 1 & 3 & 2 & & 1 & & 22 \\
\hline $\begin{array}{l}\text { Diauts de } \\
\text { costumbres: }\end{array}$ & & & & & & & 1 & & & 1 \\
\hline $\begin{array}{c}\operatorname{los} \\
\text { draubitica }\end{array}$ & 1 & & & & & & & & & 1 \\
\hline Melottann & & & 1 & & & & & & & 1 \\
\hline $\begin{array}{l}\text { Tragodia } \\
\text { gilartes:zil }\end{array}$ & & I & & & & & & & & 1 \\
\hline Tot:1 & 32 & 13 & 39 & 3 & 3 & 1 & 1 & 1 & 9 & 125 \\
\hline
\end{tabular}

\subsection{Relación de autores cuyas obras fueron representadas en Chiclana de la Frontera}

Componen la lista ordenada alfabéticamente 82 dramaturgos. Junto a los apellidos y nombre registramos el número o números correspondientes a sus obras según la relación de títulos que antecede. A continuación, en un primer paréntesis, cronología y lugar de nacimiento o fallecimiento, en caso de tener constancia de ellos, y en un segundo paréntesis, los años 
en que se representan sus obras, el número de ellas escritas por el autor, firmadas en colaboración con otro autor o autores, el total de obras y las puestas en escena que se efectuaron de las mismas.

1. ALCÓN, Aurelio [114] (Cádiz, 1850) (1879, 1889 / - / $1 / 1 / 3)$.

2. ASQUERINO, Eduardo [49] (Barcelona, 1826-1881) (1849/ - / $1 / 1 / 1)$.

3. ASQUERINO, Eusebio [49] (Sevilla, 1822 - Madrid, 1892) (1849 / - / $1 / 1$ / 1).

4. AZA, Vital [64, 83, 104, 106, 113] (Pola de Lena-Asturias, 1851 - Madrid, 1912) $(1879,1889 / 3 / 2 / 5 / 8)$.

5. BARRANCO, Mariano [69] (Valencia, 1850) $(1889 / 1 /$ - / $1 / 2)$.

6. BERMEJO, Ildefonso Antonio [65] (Cádiz, 1820-1892) (1879/ 1 / - / 1 / 1).

7. BLASCO, Eusebio [3, 24, 60, 75, 77, 96, 105, 125] (Zaragoza, 1844 - Madrid, 1903) $(1879,1889 / 7 / 1 / 8 / 12)$.

8. BRETÓN DE LOS HERREROS, Manuel [53, 67] (Quel - Logroño, 1796 - Madrid, 1873) $(1879 / 2 /-/ 2 / 2)$

9. BUENO DE SAUCAL, José María [37] (1879 / - / 1 / 1 / 1).

10. BURGOS, Javier [74, 79] (Puerto de Santa María - Cádiz, 1852 - Madrid, 1902) $(1889 / 2 /-/ 2 / 2)$.

11. CALVACHO, Carlos [86] (Madrid, 1834-1885) (1879, 1889/ $1 /$ - / $1 / 3)$.

12. CAMPO ARANA, José [85, 91, 121] (Madrid, 1847-1885) (1879, 1889 / - / 3 / 3 /

$3)$.

13. CANALES, Pedro [101] (Cádiz, 1870) $(1889 / 1 /$ - / $1 / 1)$.

14. CANO Y MASAS, Leopoldo [88] (Valladolid, 1844 - Madrid, 1934) (1889 / 1 / - / $1 / 2)$.

15. CAÑIZARES, José de [51] (Madrid, 1676-1750) (1806/ $1 /$ - / $1 /$-).

16. CASTILLO, Juan del [1] (1889/1/ - / / 1).

17. CASTILLO, Pelayo del [39] (Castellón, 1837 - Madrid, 1883) (1879/1/ - / 1/1).

18. CAVESTANY, Juan Antonio [42] (Sevilla, 1861-1924) (1879/ $1 /$ - / 1 / 2).

19. COELLO, Carlos [85] (Madrid, 1850 - 1888) (1889/ - / $1 / 1 / 1)$.

20. COLL, Gaspar Fernando [36, 117] (Palma de Mallorca, 1814-1855) (1879/ $1 / 1 / 2$ /2).

21. CORONA BUSTAMANTE, José [94] (1879/ - / $1 / 1 / 1)$.

22. CORZO Y BARRERA, Antonio [35] (Murió en 1897) (1879/1/ - / / 1).

23. CRUZ, Ramón de la [84, 89] (Madrid, 1731-1794) (1889 / 2 / - / 2 / 2).

24. CRUZ TIRADO Y NARIO, Juan de la [117] (Sevilla, 1812-1891) (1879 / - / 1 / 1 /

$1)$.

25. CHACEL, Mariano [59] (Salamanca, 1846-1882) (1889/1/ - / 1/1). 
26. ECHEGARAY, José [19, 43] (Madrid, 1832-1916) (1877, 1879 / 2 / - / 2 / 2).

27. ECHEGARAY, Miguel [20, 38, 40, 47, 54] (Quintanar de la Orden -Toledo, 1848 Madrid, 1927) (1879, 1889 / 5 / - / 5 / 5).

28. ESTREMERA, José [121] (Lérida, 1852 - Madrid, 1895) (1889 / - / 1 / 1 / 1).

29. FERNÁNDEZ BREMÓN, José [34] (Gerona, 1839 - Madrid, 1914) (1889 / 1 / - / 1 /2).

30. FUENTES, José de [91, 114] (1879 / - / 2 / 2 / 4).

31. GARCÍA DONCEL, Carlos [122] (Murió en Madrid en 1851) (1879/ - / 1/1/1).

32. GARCÍA GUTIÉRREZ, Antonio [10, 123] (Chiclana - Cádiz, 1813 - Madrid, 1884) $(1879,1883$ / 2 / - / 2 / 2).

33. GARCÍA SANTISTEBAN, Rafael [50] (Madrid, 1829-1893) (1889 / 1 / - / 1 / 1).

34. GIL, Isidoro (Madrid, 1814-1866) [63] (1889/ 1 / - / 1 / 1).

35. GIL DE ZÁRATE, Antonio [13] (El Escorial - Madrid, 1793 - Madrid, 1861)

(Décadas de 1830-1840/ $1 /$ - / 1 / -).

36. GIL Y LUENGO, Constantino [124] (Zaragoza, 1844-1914) (1889 / 1 / - / 1 / 1).

37. GÓMEZ DE CÁDIZ, Manuel [41] (Cádiz, 1849) (1879 / - / 1 / 1 / 1).

38. GUTIÉRREZ DE ALBA, José [27] (Alcalá de Guadaira - Sevilla, 1822-1897)

$(1879,1889 / 1 /-/ 1 / 2)$.

39. HARTZENBUSCH, Juan Eugenio [57] (Madrid, 1806-1880) (1879 / - / $1 / 1 / 1)$.

40. HUGO, Víctor [115] (Besançon, 1802 - París, 1885) (1879/1/ - / 1 / 1).

41. INFANTE DE PALACIOS, Santiago [4] (1889/ 1 / - / 1 / 2).

42. JACKSON CORTÉS, Eduardo [112] (Cádiz, 1826 - Pozuelo de Alarcón - Madrid, 1890) $(1879 / 1 /-/ 1 / 1)$.

43. JACKSON VEYAN, José [16, 100] (Cádiz, 1852 - Madrid, 1935) (1889 / 2 / - / 2 /

2).

44. LARRA, Luis Mariano de [81] (Madrid, 1830-1901) (1889/ 1 / - / 1 / 2).

45. LIERN Y CERACH, Rafael María [15, 32] (Valencia, 1833 - Madrid, 1897) (1879, $1889 / 2 /-/ 2 / 3)$.

46. LOMBÍA, Juan [71, 95, 120] (Zaragoza, 1806 - Madrid, 1851) (1879, 1889 / 3 / - / 3 / 4).

47. MATOSES, Manuel [110] (Valencia, 1844 - Madrid, 1901) (1889 / 1 / - / 1 / 1).

48. MEJÍAS Y ESCASSY, Luis [55, 108] $(1879,1889 / 2 /-/ 2 / 3)$.

49. MÍNGUEZ, Federico [56] (Madrid, 1852) (1889/ - / $1 / 1 / 1)$.

50. MORENO GIL, Pantaleón [9] (1879, 1889 / 1 / - / 1 / 2).

51. MORETO, Agustín [103] (Madrid, 1618 - Toledo, 1669) (1806/ 1 / - / 1 / -).

52. MORO MORALES, Emilio [92] (1879/ 1 / - / 1 / 1).

53. MOTA GONZÁLEZ, José [23] (Sevilla, 1836-1900) (1889 / 1 / - / 1 / 2).

54. NAVARRETE, Ramón de [30] (Madrid, - 1897) (1879/ - / $1 / 1 / 1)$. 
55. NÚÑEZ DE ARCE, Gaspar [26] (Valladolid, 1832 - Madrid, 1903) (1879 / 1 / - / 1 / $1)$.

56. OLONA, Luis [70, 73, 99] (Málaga, 1823 - Barcelona, 1863) (1877, 1879 / 3 / - / 3 / 3).

57. ORTIZ DE PINEDO, Manuel [8, 97] (Aracena - Huelva, 1830-1901) (1879, 1889 / $2 /-/ 2 / 2)$.

58. PALENCIA, Ceferino [12] (Cuenca, 1860 - Madrid, 1928) (1889/1/ - / 1 / 1).

59. PERAL, Juan del [21, 66] (Murió en 1888) (1849, $1889 / 2 /-/ 2 / 2)$.

60. PÉREZ ECHEVARRÍA, Francisco [62] (Madrid, 1842-1884) (1889 / 1 / - / 1 / 2).

61. PINA DOMÍNGUEZ, Mariano [22, 30, 45, 46, 61] (Granada, 1840 - Madrid, 1895) (1879/4 / $1 / 5 / 5)$.

62. PINA Y BOHIGAS, Mariano [102, 116] (Madrid, 1820-1880) $(1879,1889$ / 2 / - / 2 /2).

63. RAMOS CARRIÓN, Miguel [6, 11, 29, 60, 78, 83, 104] (Zamora, 1851 - Madrid, 1915) $(1879,1889 / 4 / 3 / 7 / 11)$.

64. ROSSELL, Cayetano [57] (Aravaca - Madrid, 1817 - Madrid, 1883) (1879 / - / 1 / $1 / 1)$.

65. RUBIO, Ángel [56] (1889/ - / $1 / 1 / 1)$.

66. RUIZ PELAYO, Samuel [90] (1883 / 1 / - / 1 / 1).

67. SÁNCHEZ ALBARRÁN, José [48] (Cádiz, 1825-1883) (1879/ 1 / - / 1 / 1).

68. SÁNCHEZ DE CASTILLA, Eduardo [7, 41, 107] (¿Cádiz? - 1899) (1879, 1889 / 2 / $1 / 3 / 3)$.

69. SÁNCHEZ GARAY, Laureano [37] (Madrid, 1824-1903) (1879 / - / $1 / 1$ / 1).

70. SANZ PÉREZ, José [17, 68, 87] (Cádiz, 1818-1870) (1879, 1889 / 3 / - / 3 / 4).

71. SELLÉS, Eugenio [80] (Granada, 1842 - Madrid, 1926) (1879/ 1 / - / 1 / 1).

72. SERRA, Narciso [52] (Madrid, 1830-1877) (1879/1/ - / $1 / 1)$.

73. TRIGO, Carlos [76] (1879/1/ - / 1/1).

74. VALLADARES GARRIGA, Luis [57, 122] (Falleció en Madrid en 1856) (1879 / - / $2 / 2 / 2$ ).

75. VALLADARES Y SAAVEDRA, Ramón de [5, 118] (Algeciras - Cádiz, 1824-1901) $(1879,1889 / 2 /-/ 2 / 3)$.

76. VEGA, Ricardo de la [93] (Madrid, 1839-1910) (1879/1/ - / 1 / 1).

77. VEGA, Ventura de la [2] (Buenos Aires - Argentina, 1807 - Madrid, 1865) (1889/ $1 /-/ 1 / 1)$.

78. VEGA Y CARPIO, Félix Lope de [72] (Madrid, 1562-1635) (1806 / 1 / - / 1 / -).

79. VILLA Y VALLE, J. de la [94] (1879/ - / $1 / 1 / 1)$.

80. ZAMORA CABALLERO, Eduardo [25] (Valencia, 1835 - Madrid, 1899) (1889/ 1 / - / 1/1). 
81. ZORRILLA, José [119] (Valladolid, 1817 - Madrid, 1893) (1849 / 1 / - / 1 / 1).

82. ZUMEL, Enrique [28, 82] (1822 - Murió en Madrid en 1897) (1879 / 2 / - / 2 / 2).

No conocemos los autores de las obras D. Ricardo y D. Ramón, Dos contra uno o Los enredos de una viuda, La feria de San Antonio en Chiclana, ¡Ladrones! ¡Ladrones!, El premio del bien, Será este y La sombra de la venganza.

\begin{tabular}{|c|c|c|}
\hline \multicolumn{3}{|c|}{$\begin{array}{c}\text { Autores por número de representaciones y de obras } \\
\text { puestas en escena }\end{array}$} \\
\hline $\begin{array}{c}\text { N. }{ }^{\circ} \text { Representaciones } \\
\text { u obras }\end{array}$ & $\begin{array}{c}\text { Autores, 82 / N. } \\
\text { representaciones }\end{array}$ & $\begin{array}{c}\text { Autores, 82 / N. } \\
\text { obras presentadas }\end{array}$ \\
\hline Sin determinar & 4 & 62 \\
\hline 1 & 43 & 10 \\
\hline 2 & 19 & 5 \\
\hline 3 & 9 & 3 \\
\hline 4 & 2 & 1 \\
\hline 5 & 2 & 1 \\
\hline 7 & 1 & \\
\hline 8 & 1 & \\
\hline 11 & 1 & \\
\hline 12 & & \\
\hline
\end{tabular}




\section{COMPAÑIIAS Y ACTORES. RECEPCIÓN CRÍTICA. SOLICITUDES DE LICENCIA PARA ACTUAR EN CHICLANA DE LA FRONTERA}

En Chiclana actuaron 9 compañías y 1 agrupación de aficionados a lo largo del siglo XIX en los años 1849, 1850, 1877, 1879, 4, 1883 y 1889, 2, al tiempo que se produjeron 9 peticiones de licencia ante las autoridades para presentarse en la ciudad por parte de otros tantos autores o directores de compañías, que no sabemos si llevaron a cabo representaciones, en 1813, $1822,1836,1850,3,1851,2$, y 1852 . También nos consta la presencia de una compañía, en 1856, a la que se anula una función el día 28 de agosto por no haber solicitado el correspondiente permiso del Ayuntamiento, y del actor Paulino Delgado en una fecha sin determinar a finales de siglo.

\begin{tabular}{|c|c|c|}
\hline \multicolumn{3}{|c|}{$\begin{array}{c}\text { Compañías que actúan en Chiclana y solicitudes de licencia para } \\
\text { presentarse en la ciudad }\end{array}$} \\
\hline Compañía & $\begin{array}{c}\text { Fecha de actuación } \\
\text { y n. }{ }^{\circ} \text { de funciones }\end{array}$ & $\begin{array}{c}\text { Solicitud de } \\
\text { licencia }\end{array}$ \\
\hline $\begin{array}{c}\text { Cía. Cómica de Cantado } \\
\text { y Baile Nacional, autor } \\
\text { Vicente Torretagle }\end{array}$ & & 1813,6 de mayo \\
\hline Cía. Nicolás Muñoz & & 1822,23 de febrero \\
\hline Cía. con José León & & 1836, abril \\
\hline Cía. Dramática, Sr. Torromé & 1849, junio, 2 f. & \\
\hline $\begin{array}{c}\text { Cía. ambulante dramática, } \\
\text { autor Francisco Cortés y } \\
\text { Flores }\end{array}$ & & 1850,31 de enero, \\
\hline $\begin{array}{c}\text { Cía. ambulante, } \\
\text { representante Cayetano } \\
\text { Rodríguez }\end{array}$ & & 1850, junio \\
\hline
\end{tabular}




\begin{tabular}{|c|c|c|}
\hline $\begin{array}{l}\text { Cía. Dramática dirigida por } \\
\text { Fernando González }\end{array}$ & $\begin{array}{c}\text { 1850, agosto, } \\
\text { septiembre, } 3 \mathrm{f} .\end{array}$ & \\
\hline Cía. ambulante, José Corte & & 1851, junio \\
\hline $\begin{array}{l}\text { Cía. dirigida por Fernando } \\
\text { González }\end{array}$ & & 1851, agosto \\
\hline $\begin{array}{l}\text { Cía. ambulante, autor } \\
\text { Manuel Brabo }\end{array}$ & & 1852, marzo \\
\hline Cía. lírico-dramática & $\begin{array}{c}\text { 1856, } 28 \text { de agosto, } \\
\text { anulada. }\end{array}$ & \\
\hline $\begin{array}{c}\text { Cía. dirigida por Carlos } \\
\text { Barrilaro del Valle }\end{array}$ & 1877, julio, $1 \mathrm{f}$. & \\
\hline $\begin{array}{l}\text { Cía. Dramática dirigida por } \\
\text { Carlos Barrilaro del Valle }\end{array}$ & $\begin{array}{c}\text { 1879, mayo, junio, } \\
13 \mathrm{f} .\end{array}$ & \\
\hline Cía. Dramática Albarrán & $\begin{array}{l}\text { 1879, julio, agosto, } \\
18 \mathrm{f} .\end{array}$ & \\
\hline Cía. Dramática & 1879, agosto, $2 \mathrm{f}$. & \\
\hline Cía. Cómico-dramática & $\begin{array}{l}\text { 1879, septiembre, } \\
\text { octubre, } 6 \mathrm{f} .\end{array}$ & \\
\hline $\begin{array}{c}\text { Sociedad García Gutiérrez, } \\
\text { aficionados }\end{array}$ & $1883,1 \mathrm{f}$. & \\
\hline $\begin{array}{c}\text { Cía. Cómico-dramática } \\
\text { dirigida por José María } \\
\text { López }\end{array}$ & $\begin{array}{l}\text { 1889, agosto, } \\
\text { Septiembre, } \\
\text { octubre, } 33 \mathrm{f} .\end{array}$ & \\
\hline Nueva sociedad & 1889 , octubre, $1 \mathrm{f}$. & \\
\hline $\begin{array}{c}\text { Cía. con el actor Paulino } \\
\text { Delgado }\end{array}$ & $\begin{array}{c}\text { Finales de } \\
\text { siglo, fecha sin } \\
\text { determinar. } \\
\end{array}$ & \\
\hline
\end{tabular}

Los días 13 y 15 de junio de 1849 durante la feria de San Antonio se presenta en la Casa teatro la Compañía dramática del Sr. Torromé. Las 
críticas aparecidas en El Liro solo citan al propio Torromé, la Sra. Guerra y el Sr. Carbia. La primera noche en Traidor, inconfeso y mártir hubo de todo. El Sr. Torromé, actor ya conocido del público:

[...] estuvo bien feliz y supo retratarnos con bastante exactitud el carácter reservado y misterioso del protagonista Gabriel. Mucho ha adelantado este actor, y si continúa con la misma aplicación en el difícil arte que ha abrazado, le auguramos un lisonjero porvenir. El público supo apreciar su trabajo, y así se lo demostró con repetidos aplausos. La Sra. Guerra, encargada del papel de Aurora y el Sr. Carbia de el de capitán Santillana, cumplieron por su parte a nuestra satisfacción y a la del público todo. Aconsejamos a este joven actor que no se precipite tanto y que modere su acción; efecto de la precipitación que sin duda es hija del entusiasmo, le faltó la voz en la escena en que pinta a Aurora su amor. Los demás actores hicieron lo que pudieron, y sentimos mucho no poder decir de ellos lo mismo que de los que ya dejamos citados.

En la pieza Un cuarto con dos camas, “[... l la ejecución fue regular por parte del Sr. Torromé, no así por la del otro actor que le acompañó".

En la función del día 15 el drama Guerras civiles tuvo una interpretación "[...] regular por parte de los Sres. Torromé y Carbia y de la Sra. Guerra. Los demás actores hicieron cuanto les fue dable por complacer al público [...]"; y en Dos contra uno o Los enredos de una viuda la actuación fue bastante regular (El Liro, 10-06-1849, n. ${ }^{\circ} 2$; 17-061849, n. ${ }^{\text {o } 3) .}$

Fernando González, galán, autor y director de una Compañía dramática compuesta por 28 miembros, hizo uso de una licencia que se le concede en agosto de 1850 para actuar en Chiclana durante 20 días por tres representaciones, marchándose después a Jerez de la Frontera9 ${ }^{9}$. Por

${ }^{9}$ A.H.P.C., 1.3.8. Gobierno Civil, 2.4.32.caja 156, exp. 53. Fernando González solici- 
su parte el actor Carlos Barrilaro del Valle recala en la localidad en julio de 1877 al frente de una Compañía con la que trabaja al menos por una función el día $29^{10}$.

Durante la temporada en torno al verano de 1879, desde mayo a octubre, se sucede la actuación de cuatro compañías. Carlos Barrilaro del Valle regresa como primer actor y director de una Compañía dramática formada por 7 actores, 7 actrices y 2 apuntadores, que pisan las tablas en 13 ocasiones:

Primera actriz, Victoria Cabello de Barrilaro; dama joven, Amalia Calle; primera graciosa, María Calle; característica, Josefa González; otra graciosa, Micaela Hernández; actrices, Enriqueta Pérez y Juana García. Primer actor, Carlos Barrilaro del Valle; otro primer actor y segundo galán, Ángel León; galán joven, Manuel Estrada; actor de carácter, Luis Infante; característico, Francisco de la Huerta; actores, Antonio Capriles y Juan Sánchez; apuntadores, Manuel Cabello y Juan Fernández.

Tras esta, la Compañía dramática Albarrán ofrece 18 representaciones durante los meses de julio y agosto, y otras dos compañías, Dramática y Cómico-dramática, interpretan dos funciones los días 28 y 31 de agosto y seis funciones entre el 1 de septiembre y el 5 de octubre, respectivamente ${ }^{11}$.

En el año 1883 la agrupación de aficionados Sociedad García

ta permiso para dar funciones dramáticas en Chiclana de la Frontera. 26.08.1850 06.09.1850.

${ }^{10}$ A.H.M.Ch. Secretaría. Registro, Correspondencia y Comunicaciones. Signatura 199. Función del domingo 29 de julio de 1877.

${ }^{11}$ A.H.M.Ch. Cultura. Programas de mano del Teatro de Chiclana.

A.H.M.Ch. Cultura. Signatura 858. Nota de las funciones de teatro. 1879. No sabemos si al frente de la Compañía Albarrán podría estar el conocido actor y autor gaditano José Sánchez Albarrán (1825-1883). 
Gutiérrez, con motivo del 70 aniversario del nacimiento del dramaturgo chiclanero en honor del cual recibe su nombre, prepara un Programa de fiestas. Entre otras actividades, en una fecha que no podemos concretar, organiza una velada presidida por el Ayuntamiento en el Teatro de Chiclana cuyos ingresos se destinan a sufragar los gastos ocasionados por las celebraciones y el coste de una lápida que en homenaje al autor de $E l$ Trovador se coloca en la fachada de su casa natal, calle Niño Jesús, n. ${ }^{\circ}$ 13, actual Corredera Alta (El Trovador, 26-09-1932, n. ${ }^{\circ}$ 20. Inesperado hallazgo $)^{12}$.

La Compañía Cómico-dramática, dirigida por el primer actor José María López, protagoniza 33 funciones entre los días 24 de agosto y 23 de octubre de 1889. La componen 7 actrices, 10 actores y 2 apuntadores:

Primera actriz, Sebastiana Suárez; primera actriz cómica, Josefa Álvarez; primera dama joven, Pastora Leal; primera característica, Josefa Cruz de Corte; actrices, Enriqueta García, Almerinda Sánchez y Eugenia Santos.

Primer actor, José María López; primer actor cómico, Andrés Aranda; segundo galán, Manuel Garrido; galán joven, Francisco Gutiérrez; primer actor de carácter, José Bedoya; segundo galán joven, Antonio Offerál; actores, José Campos, Alejandro González, Saturnino García y Enrique Tantarelli; apuntadores, Antonio Sánchez y Manuel Campos.

Se disuelve al finalizar sus representaciones constituyéndose una Nueva Sociedad a partir de ella que solo actúa el día 27 de octubre.

Los miembros de la compañía disfrutaron de cuatro veladas en su

\footnotetext{
${ }^{12} \mathrm{El}$ título de la crónica hace referencia al hecho de que al retirar para su limpieza la lápida colocada en 1883 se encontró debajo el Programa de fiestas aludido, dejado allí por la Sociedad García Gutiérrez.
} 
beneficio. Desde los programas de mano se dirigen al público para que les favorezca con su asistencia y ponderan los atractivos de las funciones que van a interpretar. Andrés Aranda y su esposa Sebastiana Suárez de Aranda por ser muy variado el cartel que ofrecen para el día 18 de septiembre. Josefa Álvarez y Teodoro Ruíz destacan la puesta en escena de El pilluelo de París llegado el día de su beneficio, 2 de octubre:

[...] las culminantes situaciones dramáticas que encierra, unido a los muchos chistes cultos que la adornan, han conseguido que en cuantos públicos ha sido representada sea su ejecución una ovación constante para los actores a la par que grato deleite para los espectadores.

Josefa Cruz de Corte y José Bedoya elogian a los autores que van a llevar a escena una semana después, el día 9, Vital Aza y Javier de Burgos, y con cada entrada se entrega un número para participar en el sorteo de un pañuelo de espuma para señora y un alfiler de corbata para caballero. Y por último el segundo galán joven Antonio Sánchez Offerráll y el apuntador Antonio Sánchez Esquinaldo al tiempo que despliegan su ingenio poético y sortean una pava para llamar la atención del respetable y que acuda al teatro la noche del 16 de octubre, señalan la necesidad por parte de los actores de obtener unos ingresos extra ${ }^{13}$.

\footnotetext{
${ }^{13}$ A.H.M.Ch. Cultura. Programas de mano del Teatro de Chiclana. Reproducimos el poema publicado por Antonio Offerráll y Antonio Sánchez:

AL PÚBLICO

En el lenguaje teatral / decir beneficio indica, / que el agraciado publica / que no tiene ni un real. / La necesidad no asalta / nuestra bolsa hasta ese punto / pero... para cierto asunto / DINERO nos hace falta. / Esta razón imperiosa / nadie habrá que la reproche: / e invitamos esta noche / a ver la función famosa. / ¿No será un dolor por cierto / si tras esta invitación / y con tan buena función / está el teatro desierto? / Conque no dejéis de ir / prontito a tomar la entrada / que después al concluir... / nadie ya pedirá nada. / Y que ya ningún patricio / falte a la cita, tocayos, / en burro, a pie, a caballo... / i todo el mundo al beneficio!! / Que no deje de venir: / aunque sea el mundo entero / que no se duerma Montero / en aquel chiribitil.
} 
Como llevamos expuesto, la documentación analizada solo nos permite confirmar la actuación de 9 compañías en Chiclana durante el siglo XIX, pero también nos informa sobre 9 solicitudes de permiso ante el Gobierno Civil de la Provincia para dar funciones en la ciudad.

Vicente Torretagle, autor de una Compañía Cómica de Cantado y Baile Nacional que sirve en el teatro de la Ysla de León, San Fernando, se determina a veranear en Chiclana todo aquel tiempo que sus intereses le permitan y pide a 6 de mayo de 1813 el superior permiso de las autoridades. Se trata de la troupe más numerosa de cuantas se anuncia la posibilidad de trabajar en la villa:

Señoras actrices: Ramona Martínez, de Cádiz; Francisca Pérez (suple a la 1'), de Málaga; Josefa Barles, de Sevilla; María Extremera, de La Coruña; María González, de Cádiz; Cipriana Chasé, de Málaga; Ana Herrera, de Cádiz. Sobresalienta general, Dolores Murier, de Málaga.

Señores actores: Toribio Miranda, de Málaga; José Segura (suple al 1\%), de Cádiz; José Ojeda, de Cádiz; Manuel Extremera, de La Coruña; Luis Mesa, de Mallorca.

Sobresaliente general, Ramón Rosquillas, de Málaga.

Carácter anciano: Vicente Torretagle, de Málaga; Francisco Murier, de Málaga.

Carácter jocoso: José Extremera, de Cádiz; José Ximenez, de Granada; Luis Murier, de Málaga.

Apuntadores: Manuel Sierra, de Sevilla; Francisco Suárez, de la Isla; Luis Mesa, de Mallorca.

Maquinistas: José Ximénez; Antonio López. Guardarropa: Antonio Escalante.

Compañia de Ópera: Damiana Nidaura; María Extremera; Josefa Barles; Francisca Pérez; María González; Cipriana Chasé; José Segura; José Ojeda; Luis Mesa; Luis Murier; Vicente Torretagle; José Ximenez. 
Baile: Esperanza Fuentes; María González; Francisco Moreti.

Maestro de música y compositor: Antonio Pérez. Primer violín de orquesta: $N$. Llordi.

Pintores: José Ximénez; Luis Murier. Cobrador principal: Juan Pérez.

Orquesta completa.

Para el servicio de la escena: doce asistencias; dos comparsas primeras.

Nota. Todos los individuos de que se compone esta compañía tienen obligación de suplirse sucesivamente ${ }^{14}$.

En 1822 Nicolás Muñoz, de ejercicio cómico, forma una compañía en conformidad con el propietario de la Casa teatro con la intención de presentarse el día de Pascua de Resurrección y continuar la temporada "[...] hasta que el pueblo no de suficientes entradas para la manutención de los Actores", por lo que suplica se conceda el correspondiente permiso ${ }^{15}$.

De José León, vecino de Cádiz, se certifica que es persona de buena conducta cuando pide en abril de 1836 autorización para pasar a Chiclana a hacer algunas representaciones de verso en unión de sus compañeros ${ }^{16}$.

A Francisco Cortés y Flores, autor y actor de una compañía ambulante dramática, se le adjudica licencia por 30 días el 31 de enero de 1850. Sin embargo, no pudo hacer uso de ella, por lo que volvió a solicitar otra al Gobierno de la Provincia, prorrogándose la anterior por 30 días a partir del 30 de marzo. La compañía estaba integrada por Don Manuel Butrón, primer actor; Eladio Lagos, $2^{\circ}$; Diego Campos, $2^{\circ}$ joven; Francisco Corte, barba; Rafael Pereira, Francisco Ortega, Cayetano Rodríguez, José Morales, Manuel Zamora; y las actrices Doña Esperanza Cabezas, María

\footnotetext{
${ }^{14}$ A.H.M.Ch. Secretaría. Certificados, Instancias y Expedientes Varios. Signatura 357. Compañía de Vicente Torretagle.

${ }^{15}$ A.H.M.Ch. Secretaría. Certificados, Instancias y Expedientes Varios. Signatura 357.

${ }^{16}$ A.H.P.C, 1.3.8 Gobierno Civil, 2.4.32, caja 154, exp. 5. José León solicita autorización para dar funciones dramáticas en Chiclana de la Frontera. 06.05.1836-07.05.1836.
} 
Butrón, Aurora la Puente y Dolores Sánchez ${ }^{17}$.

Otra Compañía ambulante representada por el actor dramático Cayetano Rodríguez obtiene permiso por un mes, a principios de junio de 1850, para actuar en la villa del Iro. Viene de presentarse en Puerto Real y cuenta con el primer actor Antonio Calle y la primera actriz María Salado $^{18}$.

José Corte hace presente en junio de 1851 "que reunidos algunos individuos sin colocación, actores dramáticos, y siéndoles preciso buscar su subsistencia y las de sus familias", ruegan se les permita trabajar como compañía ambulante:

Actores: José Corte, Eduardo Cortés, Sebastián Vechio, Eusebio Carbajal, José Perara, José Valero, Antonio Cordoncillo, Pedro Vela, Tomás Cao, Felipe Carbajal.

Actrices: Francisca Rodríguez, Josefa Cruz, María Barba, Dolores Vázquez, Josefa Rico, Dolores Carbajal, Antonia Carbajal, Carolina Carbajal.

Cuerpo coreográfico: José Martínez, Tomás Cao, Carmen Villegas, Rosario Rin.

Consuetas: José Govea, Fernando Navarrete, Felipe Carbajal.

Fernando González, que ya había pasado por Chiclana en el verano de 1850, vuelve a conseguir licencia por treinta días en agosto de 1851, aunque en esta ocasión ignoramos si llegó a utilizarla. Adjuntaba a su petición la lista de individuos que componían la Sociedad:

\footnotetext{
${ }^{17}$ A.H.P.C, 1.3.8. Gobierno Civil, 2.4.32, caja 157, exp. 16. Francisco Cortés y Flores solicita licencia para dar funciones en Puerto Real y en Chiclana de la Frontera. 1850. ${ }^{18}$ A.H.P.C, 1.3.8. Gobierno Civil, 2.4.32, caja 156, exp. 67. Cayetano Rodríguez pide licencia para dar funciones dramáticas en Chiclana de la Frontera. 25.05.1850 - 05.06.1850.
} 
Actores. - Fernando González, Francisco Careto, Emilio Zafrané, Benito Ochart, José Del Castillo, José Bodiño, Fernando Cabrera, Eusebio Carbajal, Felipe Carbajal, José Carrero y Pedro Navarro.

Actrices. - Dolores León, Alfonsa Badía, Micaela Narváez, María Barba, Pilar Zafrané y Carolina Carvajal ${ }^{19}$.

Para finalizar, Manuel Brabo como autor de una compañía ambulante logra licencia por 30 días en marzo de 1852 para dar funciones dramáticas ${ }^{20}$.

Completan las reseñas sobre compañías la suspensión de una función anunciada para la noche del 28 de agosto de 1856 por una troupe cómico-lírica procedente de Jerez "[...] sin conocimiento ni permiso de la autoridad, ...no imponiendo al representante multa alguna por ser la primera falta" "21; y la publicación de un poema en La Semana, con motivo de la llegada a la ciudad en 1931 de la Compañía de Dramas y Comedias dirigida por los actores Julia Delgado Caro y Luis Martínez de Tovar, en el que se rememora la actuación del eminente actor Paulino Delgado, padre de la actriz, en el teatro viejo, a finales del siglo XIX (La Semana, 02-021931, n. ${ }^{\circ}$ 19. Coplas sin música ${ }^{22}$.

\footnotetext{
${ }^{19}$ A.H.P.C, 1.3.8. Gobierno Civil, 2.4.32. caja 159, exp. 2. José Corte y otros actores dramáticos solicitan licencia para dar funciones en el teatro de Chiclana de la Frontera. 26.06.1851 - 23.01.1853.

${ }^{20}$ A.H.P.C., 1.3.8. Gobierno Civil, 2.4.32, caja 159, exp. 36. Manuel Brabo pide permiso para dar funciones dramáticas en Chiclana de la Frontera. 03.03.1852 - 05.03.1852.

${ }^{21}$ A.H.P.C., 1.3.8. Gobierno Civil, 2.4.32, caja 161, exp. 65.

${ }^{22}$ EN EL TEATRO

Le dije: ¿a ti no te suena / esta actriz Delgado-Caro? / hasta puede ser la hija, / de D. Paulino Delgado. I De aquel actor eminente / que hace ya cuarenta años / que vino al teatro viejo / celebrado por ti tanto. / Su señora era la actriz / doña Alejandrina Caro, / ¿será posible que sea? / pues nada tiene de extraño. / Que siendo actores sus padres / la hija haya heredado / todo el arte que tenían; / pronto se sabrá, tocayo. / EL DUENDE.
} 


\section{RECEPCIÓN CRÍTICA DE LAS OBRAS Y AUTORES LLEVADOS A ESCENA}

Los comentarios sobre las obras puestas en escena en Chiclana se limitan a varias críticas aparecidas en El Liro, en junio de 1849, cuando actuaba en el Teatro de la villa la Compañía Dramática dirigida por el Sr. Torromé. Tres de las piezas presentadas son objeto de un breve análisis. Del drama Traidor, inconfeso y mártir de José Zorrilla se opina que:

[...] Los caracteres en él están bien sostenidos, particularmente el de Gabriel. Abunda en lindos y vivísimos diálogos y en cuanto al verso en general es excelente. No creemos sin embargo que estuvo muy feliz su célebre autor en el final; otros dramas tiene mucho mejor acabados, y en verdad que es lástima, que la última escena, la cual sin duda alguna es de las más interesantes, haga desvanecer al espectador la impresión que le produce la muerte de Gabriel, suceso principal y de más efecto en toda la obra, y en el que a nuestro entender debió concluirla su autor.

Por demás está el decir, que nuestro parecer en el particular vale bien poco, y que convenimos en un todo con los ilustrados críticos que han dicho, que en el Sr. Zorrilla los defectos son bellezas, pues su brillante genio los hace pasar desapercibidos.

El argumento de Guerras civiles, drama de Eduardo y Eusebio Asquerino, resulta:

[...] interesante y el verso dulcísimo y armonioso; particularmente el sueño y el delirio, que tiene la madre de Luis y Carlos en el último acto, son de los pasajes más poéticos y de más brillante versificación que hemos oído. 
Por el contrario, la pieza Dos contra uno o Los enredos de una viuda no gusta al gacetillero de El Liro:

[...] en verdad que nada hubiéramos perdido con no verla. Por desgracia cuenta nuestro teatro en su moderno repertorio algunas producciones inmorales; pero creemos imposible hay una sola que iguale a esta de que nos ocupamos. ¿Qué escarnio tan bajo e indecente se hace en ella de la fe conyugal!, una de las virtudes más precisas de la sociedad y acaso la primera. Semejantes obras deshonran a sus autores y ahuyentan de los teatros en que se ejecutan a las personas sensatas (El Liro, 17-06-1849, n. ${ }^{\circ} 3$ ).

En términos más de publicidad que de crítica, desde los programas de mano de las funciones efectuadas durante las temporadas de mayojunio de 1879 y de agosto-octubre de 1889 se elogian algunas de las obras representadas y a sus respectivos autores. Es el caso de El nudo gordiano de Eugenio Sellés:

La prensa de Madrid, como igualmente la de provincias, han dicho, que el Sr. Sellés ha hecho una revolución, tanto en la escena dramática, como en la sociedad; llegando al extremo, algunos periódicos, de decir, que en las próximas Cortes se presentará, por los Diputados, una proposición, para hacer una nueva Ley sobre el adulterio, argumento en que está basada esta incomparable obra.

Sobre la comedia de Miguel Echegaray Contra viento y marea se apunta que surgieron dudas con respecto a una de las escenas de cara a su programación en el Teatro de la Comedia de Madrid: 
[...] Hubo diversidad de opinión entre el autor y la dirección del teatro, y se sometió a un jurado compuesto de los Sres. D. Luis Fernández Guerra, D. Florencio Romea y el Sr. Echegaray, hermano del autor. Este jurado dio su opinión favorable al poeta, y se puso en escena, asistiendo aquella noche al teatro todos los escritores, artistas, críticos y demás personas competentes; y el resultado fue, coronar al autor y representarse 43 noches consecutivas.

Se asegura que El sombrero de copa de Vital Aza es la comedia que más éxito ha alcanzado en todos los teatros de las principales capitales, 90 representaciones en Madrid y “[...] obtiene cada día más renombre en el repertorio festivo con que cuenta nuestro teatro". En los carteles se incluyen frases como ¡VIVA EL TALENTO! o ¡GLORIA AL ARTE!

De suceso teatral califican la representación de iCariños que matan!!, "[...] una de las mejores obras del repertorio moderno". Su estreno en Madrid y en otros escenarios de España y del extranjero ha sido una continuada serie de grandes ovaciones para su autor Ceferino Palencia, "[...] reconocido hoy en la literatura como uno de los mejores poetas de nuestro siglo"23.

Mención especial merece la figura de Antonio García Gutiérrez a quien sus paisanos comienzan a homenajear en las últimas décadas del siglo XIX. La calle del Beneficiado, donde se ubicaba el teatro, pasa a rotularse con el nombre del esclarecido autor chiclanero en 1868 (Meléndez Butrón y Yeste Sigüenza, 2006: 39). En 1883 se desarrolla el Programa de fiestas, ya mencionado, con motivo de la colocación de una lápida en la fachada de su casa natal y unos años más tarde, en septiembre de 1889, la compañía dirigida por el primer actor José María López anuncia una FUNCIÓN DE GALA Y VELADA LITERARIA en su honor "[...] con el valioso apoyo de las autoridades de la ciudad"24.

\footnotetext{
${ }^{23}$ A.H.M.Ch. Cultura. Programas de mano del Teatro de Chiclana.

${ }^{24}$ A.H.M.Ch. Cultura. Programas de mano del Teatro de Chiclana.
} 
De otros dramaturgos u obras vinculados a Chiclana, en el programa de la función del domingo 1 de junio de 1879 se añade como nota importante el ensayo de "[...] otro drama nuevo en 1 acto, original de una persona muy conocida de la localidad, El premio del bien", llevado a escena en dos ocasiones. El día 14 de ese mismo mes se representa la pieza en 1 acto La feria de San Antonio en Chiclana, de la que tampoco se menciona el nombre de su autor, y en septiembre de 1889 se anuncia el estreno de la obra, de un escritor novel, Un verano en Chiclana.

\section{CONCLUSIONES}

Las fuentes solo nos proporcionan datos concretos sobre la realización de 80 funciones teatrales en Chiclana de la Frontera durante el siglo XIX para los años 1849, 1850, 1877, 1879, 1883 y 1889. De forma más vaga aluden a representaciones en 1806, años finales de la década de 1830 y primeros de 1840,1850 y en el paso al siglo XX.

Esta situación se explica en gran medida por la escasez de publicaciones impresas, fundamentales para hacer un seguimiento de la escena teatral decimonónica ${ }^{25}$.

Las temporadas en torno al verano de 1879 y 1889 son las que registran una mayor cantidad de funciones, 39 y 34 respectivamente. Las cifras referidas al resto de los años son meramente testimoniales.

\footnotetext{
${ }^{25}$ De hecho, nuestro mejor conocimiento de la cartelera teatral en 1849 y 1889 coincide con la disponibilidad de este tipo de fuentes. Citemos tres ejemplos de trabajos, dos tesis doctorales consultadas en SELITEN@T, Estudios sobre teatro, y una publicación, que inciden en la importancia de las fuentes impresas para el desarrollo de la investigación del teatro representado en la segunda mitad del siglo XIX. En la ciudad de Pontevedra, "El análisis de la vida escénica durante la segunda mitad del siglo XIX debe superar dos obstáculos relacionados entre sí: las características de la prensa local y su estado de conservación. El principal problema deriva de la ausencia total o parcial de publicaciones durante determinados períodos" (Ruibal Outes, 1997: 15). Para Logroño, "Las fuentes hemerográficas han sido imprescindibles en la elaboración de este estudio [...]" (Benito Argáiz, 2003: 1.945). En el caso de Jerez de la Frontera, la existencia ininterrumpida de fuentes impresas a lo largo de toda la segunda mitad del siglo XIX favorece la reconstrucción exhaustiva de la cartelera teatral (Álvarez Hortigosa, 2012: 190-191).
} 
Entre las 37 funciones de las que conocemos su hora de inicio, 2 en mayo-junio de 1879, 1 en 1883 y 34 durante los meses de agosto, septiembre y octubre de 1889, 1 comienza a las 20:00 horas, 8 levantan el telón a las 20:30 horas y 28 a las 20:45 horas.

Las representaciones de las 20:30 tienen lugar en mayo de 1879 y en septiembre de 1889. El público asiste al teatro a las 20:45 horas en agosto, septiembre y octubre de 1889. En ambos horarios las funciones se dan tanto en días entre semana como en sábados y domingos.

Tenemos constancia de los precios de las entradas al Teatro de Chiclana para las mismas funciones de las que conocemos su horario en las décadas de 1870 y 1880 salvo para la de 1883, 36 en total. En las de 1879, 11 de mayo y 6 de junio, con la Compañía de Carlos Barrilaro del Valle, se anuncian tres tipos de localidades, palcos sin entrada a 20 reales, lunetas a 3 y tablillas a 1 real. Esta es la única compañía para la que también se apuntan los precios de abono por 15 funciones.

Para las 34 funciones restantes, 33 llevadas a cabo por la Compañía de José María López y 1 por una Nueva Sociedad, en agosto, septiembre y octubre de 1889, en el mismo local, se ponen a la venta 6 tipos de localidades: plateas sin entrada, palcos sin íd., butacas sin íd., tablillas altas y bajas sin íd. y entrada general. Su precio fue de 20, 24, 4, 1 y 2 reales en 24 funciones.

Los precios bajan a 20, 24, 3, 1 y 1 , en 8 representaciones de los días $2,9,16,23$ y 30 de septiembre y 7, 14 y 21 de octubre de 1889; y a $20,16,2,1$ y 1 , el 17 y 18 de octubre de ese mismo año. Estas 10 funciones con precios más bajos se dan cuando la estación veraniega llega a su fin, intercaladas entre las más caras. Ocho de ellas tienen lugar en lunes, probablemente por tratarse de un día tras el fin de semana en que localidades más baratas podían animar la asistencia. 


\begin{tabular}{|c|c|c|c|}
\hline \multicolumn{2}{|c|}{ Precios para las 34 funciones entre los días 24-08 y 27-10 de 1889 } \\
\hline Localidades & $\begin{array}{c}\text { Precios diarios en } \\
\text { reales / 24 funciones. } \\
\text { 1889: agosto, } \\
\text { septiembre y octubre. }\end{array}$ & $\begin{array}{c}\text { Precios diarios } \\
\text { en reales / } \\
\text { funciones. } \\
\text { 1889: septiembre y } \\
\text { octubre. }\end{array}$ & $\begin{array}{c}\text { Precios } \\
\text { diarios en } \\
\text { reales / 2 } \\
\text { funciones. } \\
1889: \\
\text { octubre. }\end{array}$ \\
\hline $\begin{array}{c}\text { Plateas sin } \\
\text { entrada }\end{array}$ & 20 & 20 & 20 \\
\hline $\begin{array}{c}\text { Palcos sin } \\
\text { íd. }\end{array}$ & 24 & 24 & 16 \\
\hline $\begin{array}{c}\text { Butacas sin } \\
\text { íd. }\end{array}$ & 4 & 3 & 1 \\
\hline $\begin{array}{c}\text { Tablillas } \\
\text { altas } \\
\text { y bajas sin } \\
\text { íd. }\end{array}$ & 1 & 1 & 1 \\
\hline $\begin{array}{c}\text { Entrada } \\
\text { general }\end{array}$ & 2 & & \\
\hline
\end{tabular}

Las funciones tenían una estructura definida. Se componían de una obra larga, drama o comedia, en 2 y sobre todo en 3 actos, seguida de una pieza cómica en 1 acto. En los años 80 solían iniciarse con una sinfonía y en las efectuadas a beneficio de los miembros de las compañías se aumentaban a tres el número de obras, una comedia o drama en 2 actos y dos piezas cómicas, al tiempo que se adornaban con Intermedios por la Banda Municipal o sorteos de regalos entre el público ${ }^{26}$.

\footnotetext{
${ }^{26}$ En ciudades como Barcelona o Jerez de la Frontera se intercalaban bailes o piezas cantadas entre las obras puestas en escena (Cervelló Español, 2008: 1.422; Álvarez Hortigosa, 2012: 190). En Badajoz las funciones constaban frecuentemente de una o dos obras y ofrecían baile a la finalización de las mismas (Suárez Muñoz, 1994: 1.183).
} 
Las 125 obras anunciadas en la Cartelera pertenecen al teatro declamado. 117 son originales y 8 traducciones, arreglos o adaptaciones: 4 del francés, 1 del italiano y 3 cuyo idioma de procedencia no se especifica.

Queda ratificada al igual que en otros estudios dedicados al teatro decimonónico la variedad de calificativos aplicados a las mismas, $14^{27}$. Destaca el género cómico (comedia, caricatura, disparate humorístico, juguete, juguete cómico, pieza cómica y sainete) con 87 piezas, 69,6\% del total, seguido del dramático (drama, drama de costumbres, loa dramática y tragedia gitanesca) con 26 , un 20,8\%. Llama la atención la ausencia del género lírico.

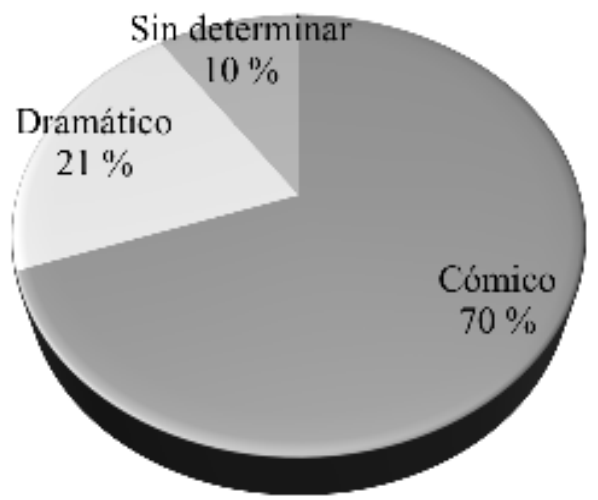

Figura 1. Porcentajes de obras puestas en escena según su género.

Las piezas en un acto, 54, suponen el 43,2\% de todas las puestas en escena por delante de las de tres actos, 39, 31,2 \% y dos, 13, 10,4 \%. Se escribieron 39 obras en verso, 23 en prosa y 2 en prosa y verso.

Se ejecutan 95 obras en una sola ocasión, de las cuales podemos

\footnotetext{
${ }^{27}$ Así quedó recogido en el ya clásico estudio de García Lorenzo (1967) y posteriormente refrendado en las carteleras de varias ciudades españolas (SELITEN@T, Estudios sobre teatro) o en el ya mencionado trabajo sobre Jerez de la Frontera (Álvarez Hortigosa, 2012: 204-210).
} 
determinar que 69 pertenecen al género cómico y 15 al dramático. De 21 obras, 11 cómicas y 10 dramas, se llevan a cabo dos representaciones, y solo 5 títulos, todos ellos comedias o juguetes, se interpretaron tres veces. Se trata de La campanilla de los apuros, ;Levantar muertos!, Para mentir las mujeres, La rosa amarilla y La sota de bastos.

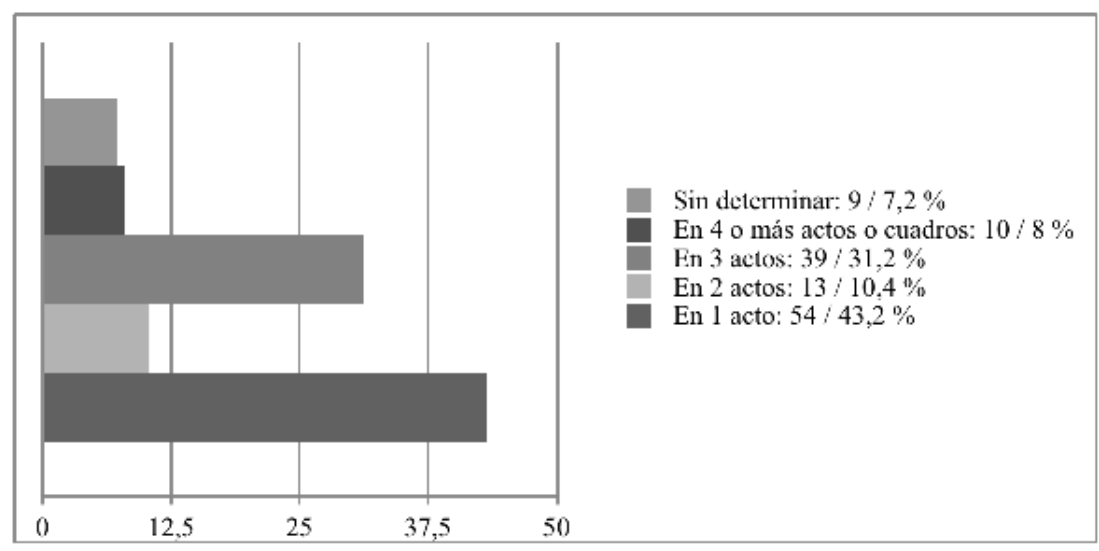

Figura 2. Porcentajes de obras según su número de actos.

Subieron al escenario las obras de 82 autores. 43 en una sola ocasión, el 52,5\%, 19 en dos, 9 en tres, 2 en cuatro, 5 en cinco o más y 4 sin determinar. Sobresalen Eusebio Blasco con 12 puestas en escena de sus obras, Miguel Ramos Carrión con 11, Vital Aza con 8, y Mariano Pina Domínguez y Miguel Echegaray con 5 cada uno.

Presentaron una obra 62 dramaturgos, el 75,6 \%, 10 dos obras, 5 tres y más de cinco Eusebio Blasco, con 8 títulos, Miguel Ramos Carrión 7, y Vital Aza, Miguel Echegaray y Mariano Pina Domínguez 5. 15 obras fueron escritas en colaboración entre dos autores y 1 entre tres.

Los autores del Siglo de Oro cuentan con dos títulos, El mejor alcalde el rey, de Lope de Vega, y El rico hombre de Alcalá, de Agustín Moreto. Suponen un 1,6 \% del total de obras escenificadas durante todo el siglo XIX. Comparado con el porcentaje de obras clásicas recogidas 
en investigaciones de otras ciudades españolas para la segunda mitad del siglo como Badajoz, 0,14 \%, Jerez de la Frontera, 0,48 \%, Las Palmas de Gran Canaria, $0,58 \%$ o León, $0,12 \%$, resulta similar y redunda en la escasa presencia del teatro áureo en la cartelera teatral decimonónica.

Ninguna de las dos obras clásicas representadas en Chiclana se puso en las otras cuatro ciudades, aunque sí sus autores. Lope subió a los escenarios de Badajoz, Jerez de la Frontera y Las Palmas de Gran Canaria con La niña boba. El desdén con el desdén, de Moreto, pasó por Jerez y Las Palmas. Calderón de la Barca fue representado en estas cuatro ciudades, Francisco de Rojas Zorrilla en Las Palmas y Jerez y Tirso de Molina y Guillén de Castro solo en Jerez ${ }^{28}$.

El teatro del siglo XVIII está presente con dos obras del popular Ramón de la Cruz, ;Los palos deseados! y El payo de la carta, y una de José de Cañizares, El honor da entendimiento.

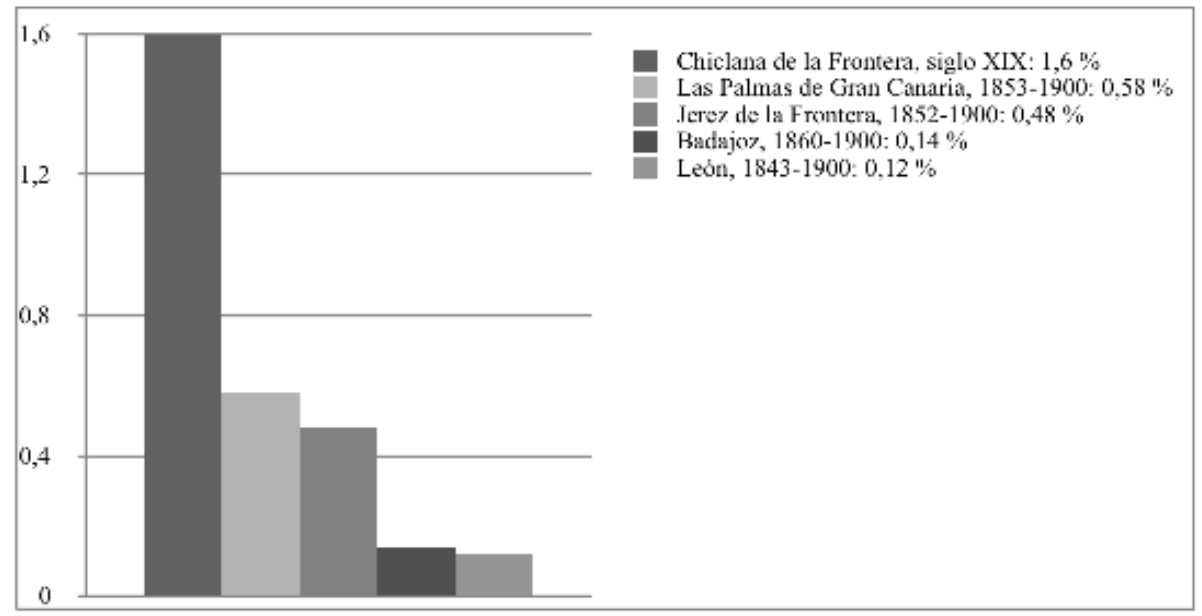

Figura 3. Porcentaje de obras del teatro clásico español representadas en cinco ciudades españolas durante distintos períodos del siglo XIX.

\footnotetext{
${ }^{28}$ En el SELITEN@T, bajo la dirección del profesor Romera Castillo, se ha desarrollado una línea de investigación con el objetivo de conocer la presencia del teatro clásico español del Siglo de Oro y del siglo XVIII en la cartelera teatral decimonónica, plasmada en la publicación de varios artículos en la revista Signa referidos a las ciudades de Las Palmas de Gran Canaria, Badajoz, León y Jerez. Del dedicado a la ciudad andaluza extraemos los datos apuntados (Álvarez Hortigosa, 2010: 183-186, 209-211).
} 
Entre los autores u obras relacionadas con Chiclana destaca la figura de Antonio García Gutiérrez y la puesta en escena de El premio del bien, original de una persona conocida de la ciudad, y La feria de Chiclana.

La mayor parte de las notas recabadas sobre la estancia de las compañías se refieren a la segunda mitad del siglo, mientras que las peticiones de licencia para actuar en la villa predominan en la primera mitad. Solo una función corrió a cargo de un grupo de aficionados, la Sociedad García Gutiérrez, en 1883.

Siete de las compañías reciben el apelativo de dramáticas, dos de cómico-dramáticas, dos incluyen el término lírico o de cantado y otras dos una sección coreográfica. A cuatro de ellas se las denomina ambulantes.

En el repertorio de las tres compañías que llevan a cabo más funciones predomina el género dramático en las 13 efectuadas en 1789 por la de Barrilaro. La de Albarrán se inclina por el cómico esa misma temporada, pues solo en 2 de las 18 funciones ejecutadas interpretan dramas. La dirigida por José María López en 1889 recurre a un programa mixto cómico-dramático en sus 33 intervenciones ante el público chiclanero, aunque más abundante en obras cómicas.

El número de componentes de las compañías oscila entre los 13 que forman la de Francisco Cortés y Flores en 1850 y los 23 incluidos en la lista presentada por José Corte de cara al mes de junio de 1851. La de Barrilaro en 1879 cuenta con 16 miembros, 17 la de Fernando González para 1851 y 19 la de José María López en 1889. Constituye una excepción la que pretende veranear en Chiclana en 1813 con Vicente Torretagle como autor ya que dispone de secciones cómica, de cantado y baile, orquesta completa y servicio de escena, superando los 40 integrantes.

Es probable que la cercanía a Cádiz favoreciera la llegada de compañías, sobre todo de cara a la temporada veraniega, si bien Chiclana no se hallaba situada entre importantes centros económicos y por añadidura teatrales, caso de Jerez, entre Cádiz y Sevilla, o León, en el trayecto de 
Madrid a Galicia y Asturias29.

También se producían desplazamientos de las compañías entre las ciudades de la bahía gaditana. De las troupes que gestionaron su paso por la ciudad, la de Torretagle servía en San Fernando, la de Cayetano Rodríguez se encontraba en Puerto Real y la compañía cómico-lírica a la que se suspendió una función en 1856 procedía de Jerez.

De alguna manera las duras condiciones de vida de los cómicos quedan reflejadas en varias de las solicitudes ya citadas para actuar en el teatro de la villa. Los actores dramáticos sin colocación reunidos por José Corte a mediados de siglo basaban su petición en la necesidad de buscar su subsistencia y la de sus familias y Nicolás Muñoz en 1822 pretendía permanecer en Chiclana hasta que el pueblo no de suficientes entradas para mantener a la compañía.

Esta realidad se veía agravada por el carácter ambulante de la ocupación de actor, adjetivo aplicado a las compañías de Francisco Cortés y Flores o de Cayetano Rodríguez en 1850, y por su negativa consideración social que se hace patente cuando se certifica la buena conducta de José León al pedir autorización en 1836 para presentarse en la ciudad30.

Por lo que respecta a la recepción crítica dispensada a los diversos elementos que constituyen el espectáculo teatral, contamos con algunas breves crónicas aparecidas en El Liro y comentarios, de carácter más publicitario que crítico, recogidos en los programas de mano de varias representaciones con el objetivo de estimular la asistencia al teatro.

Los gacetilleros fijan su atención en los actores protagonistas y las obras de algunos de los dramaturgos más valorados del momento.

\footnotetext{
${ }^{29}$ En la tesis doctoral León y su actividad escénica en la segunda mitad del siglo XIX, se describe a la ciudad como "[...] centro neurálgico del fenómeno teatral que, desde Madrid, se proyectaba hacia Galicia y Asturias” (Fernández García, 1997: 928).

${ }^{30}$ No obstante, la consideración social de los actores mejoró durante la segunda mitad del siglo XIX. "El sentido peyorativo que antes tenía el término cómico se iba desplazando paulatinamente. [...] su situación económica distaba de ser ventajosa. Tan solo los actores principales gozaron de una situación estable. En general vivían en una situación de desamparo" (Rubio Jiménez, 1998: 712-713).
} 


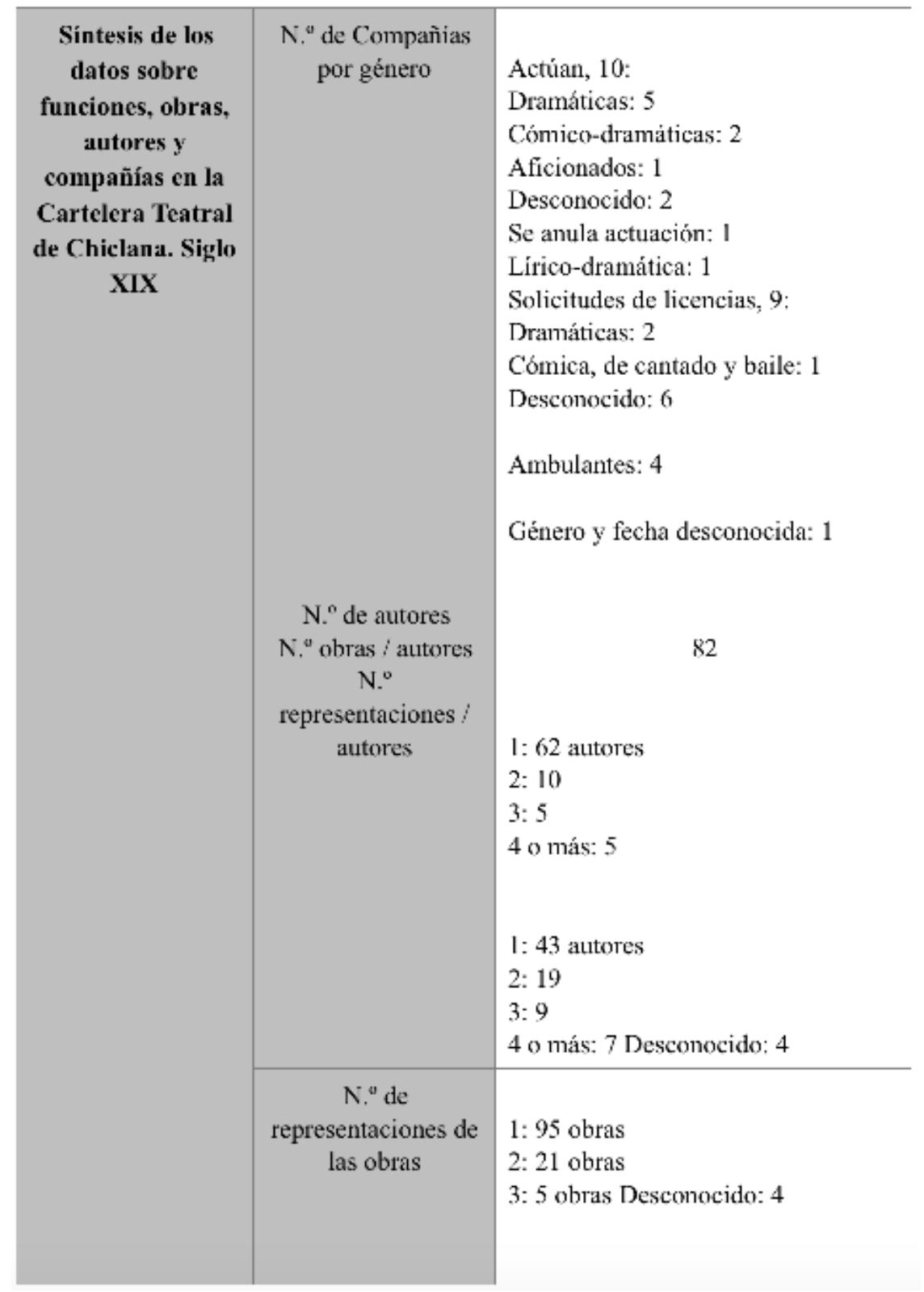




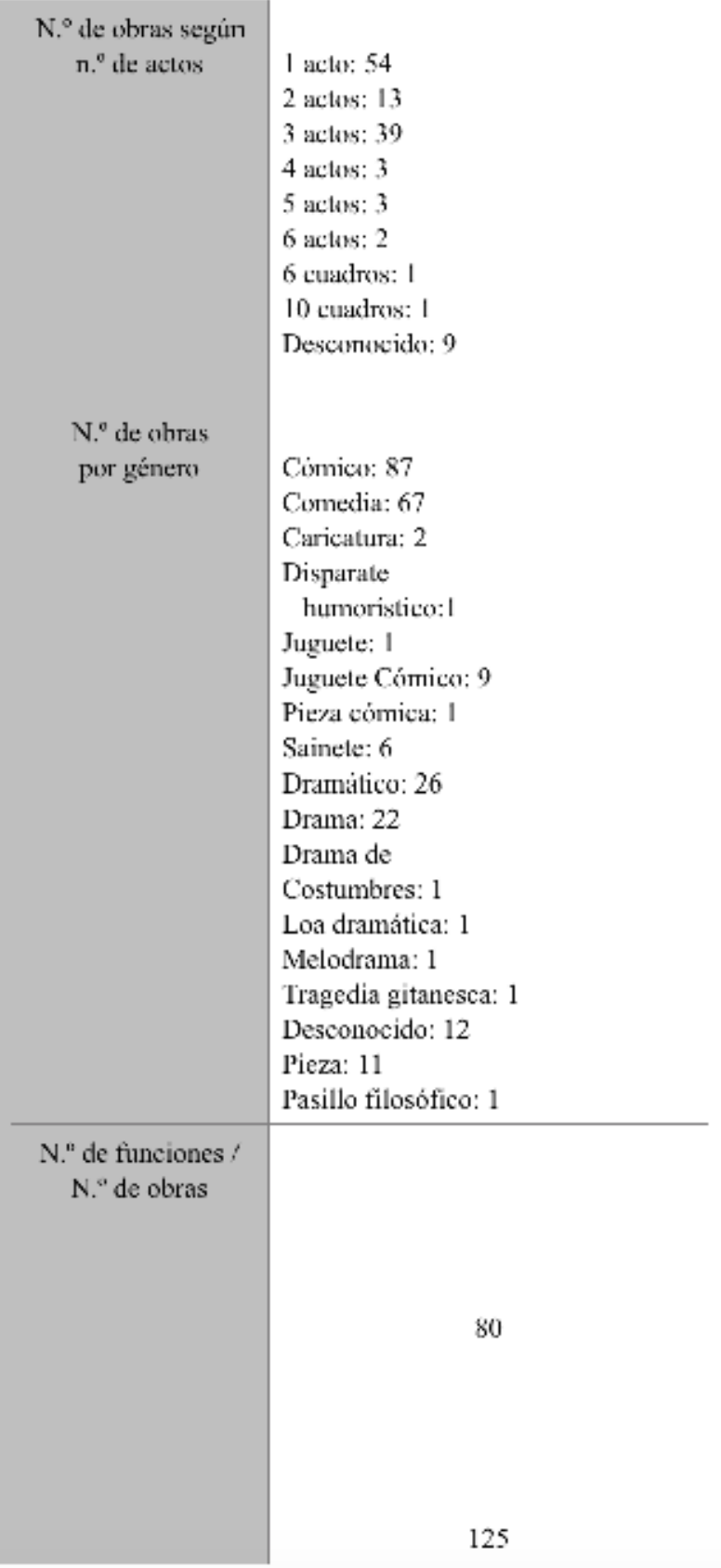




\section{REFERENCIAS BIBLIOGRÁFICAS}

ÁlVAREZ HORTIGOSA, F. (2010). "Puestas en escena del teatro clásico español del Siglo de Oro y del siglo XVIII en Jerez de la Frontera (1852-1900)". Signa. Revista de la Asociación Española de Semiótica 19, 181-212 (también en http://cervantesvirtual.com/ hemeroteca/signa [14/04/2018]).

(2012). La vida escénica en Jerez de la Frontera durante la segunda mitad del Siglo XIX. Barcelona: Anagnórisis.

BENITO ARGÁIZ, I. (2003). La vida escénica en Logroño (18501900). Tesis doctoral defendida en la Universidad de la Rioja, noviembre de 2003, bajo la dirección de M. ${ }^{a}$ Pilar Espín Templado, http://www2.uned.es/centro-investigacion-SELITEN@T/pdf/ BenitoArgaizInmaculada.pdf [14-04-2018].

BIBLIOTECA NACIONAL (1986). Catálogo del Teatro Lírico Español en la Biblioteca Nacional. I: Libretos A-CH, 1986; II: D-O, 1991; III: P-Z, 1991. Madrid: Ministerio de Cultura.

(1989). Catálogo de piezas de teatro que se conservan en el Gabinete de Manuscritos de la Biblioteca Nacional. Tomo III: Suplemento e Índices. Madrid: Ministerio de Cultura.

BOHÓRQUEZ JIMÉNEZ, D. (1996). Chiclana de la Frontera. Geografía, Historia, Urbanismo y Arte. Cádiz: Publicaciones del Sur.

CENTRO DE INVESTIGACIÓN DE SEMIÓTICA LITERARIA, TEATRAL Y NUEVAS TECNOLOGÍAS (UNED). "Estudios sobre teatro": http://www.uned.es/centro-investigación-SELITENT@/ estudios_sobre_teatro.html [14/04/2018].

CERVELLÓ ESPAÑOL, C. (2008). La vida escénica en Barcelona 18551865 (Teatro Principal y Teatro Circo Barcelonés). Tesis doctoral defendida en la UNED, febrero de 2008, bajo la dirección de M. ${ }^{a}$ 
Pilar Espín Templado, http://www2.uned.es/centro-investigacionSELITEN@T/pdf/CARLOS_CERVELL_(BARCELONA).pdf [1504-2018].

DOUGHERTY, D. y VILCHES DE FRUTOS, M. ${ }^{\text {a }}$ F. (1990). La escena madrileña entre 1918 y 1926: análisis y documentación. Madrid: Fundamentos.

FERNÁNDEZ GARCÍA, E. (1997). León y su actividad escénica en la segunda mitad del siglo XIX. Tesis doctoral defendida en la UNED, junio de 1997, bajo la dirección de José Romera Castillo, http://www2.uned.es/centro-investigacion-SELITEN@T/pdf/ estefaniafernandez.pdf [14-04-2018].

FUNDACIÓN JUAN MARCH. (1986). Catálogo de Obras de Teatro Español del siglo XIX. Madrid: Fundación Juan March.

(1993). Catálogo de Libretos Españoles. Siglos XIX y XX. Madrid: Fundación Juan March.

GARCÍA LORENZO, L. (1967). “La denominación de los géneros teatrales en España durante el siglo XIX y el primer tercio del XX”. Segismundo 5-6, 191-199.

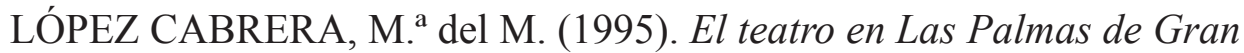
Canaria (1853-1900). Tesis doctoral defendida en la UNED, enero de 1995, bajo la dirección de José Romera Castillo, http://www2. uned.es/centro-investigacion-SELITEN@T/pdf/LopezCabrera.pdf [15-04-2018].

MADOZ, P. (1847). Diccionario Geográfico-Estadístico-Histórico de España y sus posesiones de Ultramar, tomo VII. Madrid.

MELÉNDEZ BUTRÓN, M. y YESTE SIGÜENZA, F. J. (2006). Calles y plazas de Chiclana de la Frontera (Nomenclatura histórica desde 1700). Chiclana de la Frontera: Fundación Viprén.

ROMERA CASTILLO, J. (2011). Pautas para la investigación del teatro 
español y sus puestas en escena. Madrid: UNED.

RUBIO JIMÉNEZ, J. (1998). "El teatro en el siglo XIX (1845-1900)”. En Historia del teatro en España. Tomo II, Siglo XVIII. Siglo XIX, José María Díez Borque (dir.), 625-751. Madrid: Taurus.

RUIBAL OUTES, T. (1997). La vida escénica en Pontevedra en la segunda mitad del siglo XIX. Tesis doctoral defendida en la UNED, abril de 1997, bajo la dirección de José Romera Castillo, http://www2. uned.es/centro-investigacion-SELITEN@T/pdf/RuibalOutes.pdf [14-04-2018].

SUÁREZ MUÑOZ, Á. (1994). La vida escénica en Badajoz (1860-1886). Tesis doctoral defendida en la UNED, diciembre de 1994, bajo la dirección de José Romera Castillo, http://www2.uned.es/centroinvestigacion-SELITEN@T/pdf/angelsuarez.pdf [15-04-2018].

VARGAS ZÚÑIGA, L.; ROMERO FERRER, A. y BAJO MARTÍNEZ, M. J. (2002). Catálogo de autores dramáticos andaluces, 18001897, vol. II (2 tomos). Sevilla: CDAEA.

\section{FUENTES DOCUMENTALES}

Archivo Histórico Municipal de Chiclana. A.H.M.Ch.

Secretaría. Certificados, Instancias y Expedientes Varios. Signatura 357 (1792-1822). Compañía de Vicente Torretagle, 1813. Compañía de Nicolás Muñoz, 1822.

Secretaría. Registro, Correspondencia y Comunicaciones. Signatura 199 (1875-1878). Función del domingo 29 de julio de 1877 y 201 (1884-1892). Estadística de los Edificios destinados a espectáculos públicos en esta Ciudad.

Cultura. Festejos. Expedientes anuales. Signatura 858 (1668-1959). Nota de las funciones de teatro. 1879. 
Cultura. Programas de mano del Teatro de Chiclana.

Archivo Histórico Provincial de Cádiz. A.H.P.C.

Sección Gobierno Civil diversiones y espectáculos.

1.3.8. Gobierno Civil, 2.4.32: caja 154, expedientes 5, 50; caja 156, expedientes 53,67 ; caja 157 , expediente 16 ; caja 159 , expedientes 2 , 36; caja 161, expediente 65.

Sección de Gobierno Civil. Caja 116, n. ${ }^{\circ}$ 31. Ejemplares de El Liro.

El Liro, sale el 3 de junio de 1849. De él poseemos el proyecto y los seis primeros números. Formato 21,2 por 29,7 cm., cuatro páginas, aparece los domingos. No consta ni el director ni el editor. Si sabemos que se imprimía en Chiclana, en la imprenta de don Manuel Lozano, calle Vega, 32. Entre sus secciones temas literarios y asuntos generales de la villa: empedrado, contribución, feria, teatro, etc.

Biblioteca Municipal de Chiclana. B.M.Ch.

La Semana. Periódico Independiente. Crónicas - Informaciones - Literatura. Chiclana de la Frontera. Año I, n. ${ }^{0}$ 1, 29-09-1930. Año II, desde el n. ${ }^{\circ}$ 15, 05-01-1931 al n. ${ }^{\circ}$ 30, 27-04-1931.

El Trovador. Semanario Independiente, Noticiero e Informativo. Chiclana de la Frontera. Año I, n. ${ }^{\circ}$ 1, 15-05-1932. Año II, desde el n. ${ }^{\circ} 34,02-$ 01-1933 al n. ${ }^{\circ} 81,20-11-1933$.

Recibido el 27 de enero de 2018.

Aceptado el 24 de mayo de 2018. 